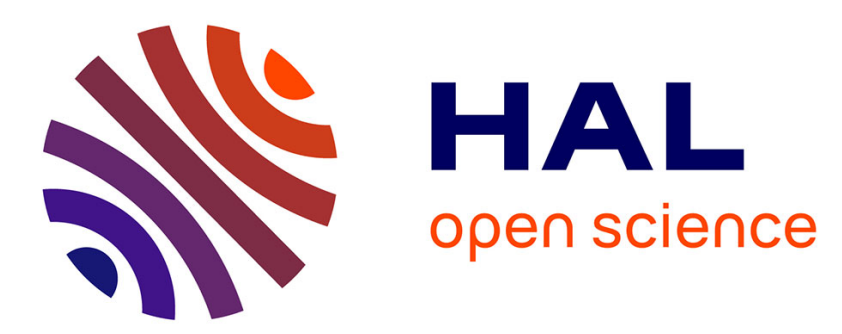

\title{
Development Of Homogeneity Concept For Time-Delay Systems
}

Denis Efimov, Wilfrid Perruquetti, Jean-Pierre Richard

\section{To cite this version:}

Denis Efimov, Wilfrid Perruquetti, Jean-Pierre Richard. Development Of Homogeneity Concept For Time-Delay Systems. SIAM Journal on Control and Optimization, 2014, 52 (3), pp.1547-1566. 10.1137/130908750 . hal-00956878

\section{HAL Id: hal-00956878 https://hal.inria.fr/hal-00956878}

Submitted on 7 Mar 2014

HAL is a multi-disciplinary open access archive for the deposit and dissemination of scientific research documents, whether they are published or not. The documents may come from teaching and research institutions in France or abroad, or from public or private research centers.
L'archive ouverte pluridisciplinaire HAL, est destinée au dépôt et à la diffusion de documents scientifiques de niveau recherche, publiés ou non, émanant des établissements d'enseignement et de recherche français ou étrangers, des laboratoires publics ou privés. 


\title{
DEVELOPMENT OF HOMOGENEITY CONCEPT FOR TIME-DELAY SYSTEMS
}

\author{
EFIMOV D., PERRUQUETTI W., RICHARD J.-P.
}

\begin{abstract}
The notion of homogeneity is extended to the time-delay nonlinear systems. Applications of Lyapunov-Krasovskii functionals and LyapunovRazumikhin functions for stability investigation are analyzed. The notion of local homogeneity is introduced, relations between stability/instability of the locally approximating dynamics and the original time-delay system are established. A link between homogeneity and input-to-state stability is investigated. Examples of application of the proposed theory are given.
\end{abstract}

\section{INTRODUCTION}

For nonlinear dynamical systems, behavior of trajectories of a homogeneous system can be extended to the whole state space based on their behavior on a suitably defined sphere around the origin [1]. Thus the state vector rescaling does not change the system behavior. As it has been shown during the last two decades this property can be used for stability analysis $[2,3,4,5,6]$, systems approximation $[7,8]$, stabilization $[9,10,11,12,13]$ and observation $[2,8]$. Analysis and synthesis of homogeneous systems is a little bit simpler, for example, the Lyapunov function has to be constructed on the unit sphere only (on the whole state space it can extended using rescaling). Moreover, it has been shown that for stability/instability analysis, Lyapunov function of a homogeneous system can be chosen homogeneous [6, 14].

In the work [2] the homogeneity in the bi-limit has been introduced, that is homogeneity with different weights and approximating functions at a vicinity of the origin and at infinity. Recently the bi-limit homogeneity has been extended to the local homogeneity [14], when the system is similar to a homogeneous one on a sphere only. In this case the homogeneous systems theory allows the original nonlinear system behavior to be analyzed locally.

This theory has been developed for continuous time-invariant nonlinear ordinary differential equations (ODEs). Another important class of systems includes those described by the differential equations with time-delayed states. The presence of

The authors are with Non-A team at Inria, Parc Scientifique de la Haute Borne, 40 avenue Halley, 59650 Villeneuve d'Ascq, France and with LAGIS (UMR-CNRS 8146), Ecole Centrale de Lille, BP 48, Cité Scientifique, 59651 Villeneuve-d'Ascq, France.

Efimov D. is with Department of Control Systems and Informatics, University ITMO, 49 avenue Kronverkskiy, 197101 Saint Petersburg, Russia. 
delays is usual in many applications $[15,16]$. Analysis of a delay influence on the system stability is critical for many natural and human-developed systems [17, 18, 19]. For instance, a large literature was recently devoted to networked systems (see $[20,21]$ and their references) which suffer from various sources of delays (such as access time, communication or packet dropouts), as well as to embedded/real-time systems, the asynchronous sampling effect of which can be modeled by delays (see [22] and the references herein). However, most of the examples in this literature are modeled by linear time-delay systems (i.e., with constant coefficients and possibly variable delays). This is due to the fact that, for such models, the stability analysis is already well developed with even converse Lyapunov-Krasovskii theorems in the case of constant delays [17]. However, for nonlinear applications, design of a Lyapunov-Krasovskii functional or a Lyapunov-Razumikhin function is still a difficult problem. A motivation for this study is that stability analysis could be (at least partially) facilitated by using homogeneity arguments, as it has been done for ODEs, where the class of homogeneous systems includes linear ones while extending some advantages of linear system theory to nonlinear domain. In this way, for instance, analysis of networking/sampling systems can be eased if the homogeneity concept can be properly extended to time-delay systems.

The goal of this work is to develop the homogeneity approach to the nonlinear time-delay systems. The problem is that delay systems operate in the infinite dimensional state space. Extension of the standard results [6] to this class of systems needs a complete revision of the homogeneous system apparatus. There exist a few works dealt with time-delay systems using the homogeneity theory [23, 24, 25, 26]. In $[24,26]$ a homogeneous (linear) delay differential equation has been studied, in [25] the cooperative and homogeneous systems have been analyzed. In all these works, the homogeneity theory has not been extended to the functional spaces and the papers are based on homogeneity of non-delayed parts of the differential equations.

The outline of this work is as follows. The preliminary definitions and the system equations are given in Section 2. The homogeneous norm, an extended definition of homogeneity for time-delay systems, as well as sufficient stability/instability conditions are presented in Section 3. The local homogeneity theory is studied in Section 4. In $[27,28,29,30]$ it has been shown that for ordinary differential equations, the homogeneity implies some kind of robustness with respect to external disturbances: a corresponding link between input-to-state stability (ISS) and homogeneity for a nonlinear time-delay system is established in Section 5. Examples are presented in Section 6 . 


\section{Preliminaries}

Consider an autonomous functional differential equation of retarded type [19]:

$$
d x(t) / d t=f\left(x_{t}\right), t \geq 0,
$$

where $x \in \mathbb{R}^{n}$ and $x_{t} \in C_{[-\tau, 0]}$ is the state function, $x_{t}(s)=x(t+s),-\tau \leq s \leq 0$ (we denote by $C_{[a, b]}, 0 \leq a<b \leq+\infty$ the Banach space of continuous functions $\phi$ : $[a, b] \rightarrow \mathbb{R}^{n}$ with the uniform norm $\|\phi\|=\sup _{a \leq \varsigma \leq b}|\phi(\varsigma)|$, where $|\cdot|$ is the standard Euclidean norm); $f: C_{[-\tau, 0]} \rightarrow \mathbb{R}^{n}$ is a locally Lipschitz continuous function, $f(0)=0$. The representation (1) includes pointwise or distributed retarded systems with either constant or variable time delay $\tau(t) \in[0, \tau]$. We assume that solutions of the system (1) satisfy the initial functional condition $x_{0} \in C_{[-\tau, 0]}$. It is known from the theory of functional differential equations [19] that under the above assumptions the system (1) has a unique solution $x\left(t, x_{0}\right)$ satisfying the initial condition $x_{0}$, which is defined on some finite time interval $[-\tau, T$ ) (we will use the notation $x(t)$ to reference $x\left(t, x_{0}\right)$ if the origin of $x_{0}$ is clear from the context).

The upper right-hand Dini derivative of a locally Lipschitz continuous functional $\mathcal{V}: C_{[-\tau, 0]} \rightarrow \mathbb{R}_{+}$along solutions of the system (1) is defined as follows for any $\phi \in C_{[-\tau, 0]}$ :

$$
D^{+} \mathcal{V}(\phi)=\lim \sup _{h \rightarrow 0^{+}} \frac{1}{h}\left[\mathcal{V}\left(\phi_{h}\right)-\mathcal{V}(\phi)\right],
$$

where $\phi_{h} \in C_{[-\tau, 0]}$ for $0<h<\tau$ is given by

$$
\phi_{h}(\theta)= \begin{cases}\phi(\theta+h), & \theta \in[-\tau,-h) \\ \phi(0)+f(\phi)(\theta+h), & \theta \in[-h, 0] .\end{cases}
$$

For a locally Lipschitz continuous function $V: \mathbb{R}^{n} \rightarrow \mathbb{R}_{+}$the lower or upper directional Dini derivatives are defined as follows:

$$
\begin{aligned}
& D^{-} V\left[x_{t}(0)\right] f\left(x_{t}\right)=\lim _{h \rightarrow 0^{+}} \inf \frac{V\left[x_{t}(0)+h f\left(x_{t}\right)\right]-V\left[x_{t}(0)\right]}{h}, \\
& D^{+} V\left[x_{t}(0)\right] f\left(x_{t}\right)=\lim _{h \rightarrow 0^{+}} \sup \frac{V\left[x_{t}(0)+h f\left(x_{t}\right)\right]-V\left[x_{t}(0)\right]}{h} .
\end{aligned}
$$

A continuous function $\sigma: \mathbb{R}_{+} \rightarrow \mathbb{R}_{+}$belongs to class $\mathcal{K}$ if it is strictly increasing and $\sigma(0)=0$; it belongs to class $\mathcal{K}_{\infty}$ if it is also radially unbounded. A continuous function $\beta: \mathbb{R}_{+} \times \mathbb{R}_{+} \rightarrow \mathbb{R}_{+}$belongs to class $\mathcal{K} \mathcal{L}$ if $\beta(\cdot, r) \in \mathcal{K}$ and $\beta(r, \cdot)$ is a strictly decreasing to zero for any fixed $r \in \mathbb{R}_{+}$. The symbol $\overline{1, m}$ is used to denote a sequence of integers $1, \ldots, m$.

\section{Homogeneity}

For any $r_{i}>0, i=\overline{1, n}$ and $\lambda>0$, define the dilation matrix $\Lambda_{r}(\lambda)=$ $\operatorname{diag}\left\{\lambda^{r_{i}}\right\}_{i=1}^{n}$ and the vector of weights $r=\left[r_{1}, \ldots, r_{n}\right]^{T}$. 
For any $r_{i}>0, i=\overline{1, n}$ and $x \in \mathbb{R}^{n}$ the homogeneous norm can be defined as follows

$$
|x|_{r}=\left(\sum_{i=1}^{n}\left|x_{i}\right|^{\rho / r_{i}}\right)^{1 / \rho}, \rho \geq \max _{1 \leq i \leq n} r_{i} .
$$

For all $x \in \mathbb{R}^{n}$, its Euclidean norm $|x|$ is related with the homogeneous one:

$$
\begin{gathered}
\underline{\sigma}_{r}\left(|x|_{r}\right) \leq|x| \leq \bar{\sigma}_{r}\left(|x|_{r}\right), \\
\bar{\sigma}_{r}(s)=\max _{|x|_{r} \leq s}|x|, \underline{\sigma}_{r}^{-1}(s)=\max _{|x| \leq s}|x|_{r},
\end{gathered}
$$

where $\underline{\sigma}_{r}, \bar{\sigma}_{r} \in \mathcal{K}_{\infty}$ define the Euclidean norm deviations with respect to the homogeneous norm. The homogeneous norm has an important property that $\left|\Lambda_{r}(\lambda) x\right|_{r}=\lambda|x|_{r}$ for all $x \in \mathbb{R}^{n}$. Define $\mathbb{S}_{r}=\left\{x \in \mathbb{R}^{n}:|x|_{r}=1\right\}$.

Since the transformation by dilation matrix $\Lambda_{r}(\lambda)$ is linear, it can be applied to functional arguments. Indeed, for any $r_{i}>0, i=\overline{1, n}$ and $\phi \in C_{[a, b]}, 0 \leq a<b \leq$ $+\infty$ the homogeneous norm can be defined as follows

$$
\|\phi\|_{r}=\left(\sum_{i=1}^{n}\left\|\phi_{i}\right\|^{\rho / r_{i}}\right)^{1 / \rho}, \rho=\prod_{i=1}^{n} r_{i} .
$$

Lemma 1. There exist two functions $\underline{\rho}_{r}, \bar{\rho}_{r} \in \mathcal{K}_{\infty}$ such that for all $\phi \in C_{[a, b]}$

$$
\underline{\rho}_{r}\left(\|\phi\|_{r}\right) \leq\|\phi\| \leq \bar{\rho}_{r}\left(\|\phi\|_{r}\right) .
$$

Proof. Let $\|\phi\|_{r} \leq s$ for some $s \in \mathbb{R}_{+}$, then by the norm definition $\left\|\phi_{i}\right\|^{\rho / r_{i}} \leq$ $\sum_{i=1}^{n}\left\|\phi_{i}\right\|^{\rho / r_{i}} \leq s^{\rho}$ and $\left\|\phi_{i}\right\|=\sup _{a \leq \varsigma \leq b}\left|\phi_{i}(\varsigma)\right| \leq s^{r_{i}}$ for each $i \in \overline{1, n}$. Therefore, $\|\phi\|=\sup _{a \leq \varsigma \leq b}|\phi(\varsigma)|=\sup _{a \leq \varsigma \leq b} \sqrt{\sum_{i=1}^{n} \phi_{i}^{2}(\varsigma)} \leq \sqrt{\sum_{i=1}^{n}\left[\sup _{a \leq \varsigma \leq b}\left|\phi_{i}(\varsigma)\right|\right]^{2}} \leq$ $\sqrt{\sum_{i=1}^{n} s^{2 r_{i}}}$. Take $\bar{\rho}_{r}(s)=\sqrt{\sum_{i=1}^{n} s^{2 r_{i}}}$, obviously $\bar{\rho}_{r} \in \mathcal{K}_{\infty}$. Inversely, let $\|\phi\| \leq s$ for some $s \in \mathbb{R}_{+}$, then $\sup _{a \leq \varsigma \leq b} \phi_{i}^{2}(\varsigma) \leq \sup _{a \leq \varsigma \leq b} \sum_{i=1}^{n} \phi_{i}^{2}(\varsigma) \leq s^{2}$ and $\left\|\phi_{i}\right\|=$ $\sup _{a \leq \varsigma \leq b}\left|\phi_{i}(\varsigma)\right| \leq s$ for each $i \in \overline{1, n}$. Finally, $\|\phi\|_{r}=\left(\sum_{i=1}^{n}\left\|\phi_{i}\right\|^{\rho / r_{i}}\right)^{1 / \rho} \leq$ $\left(\sum_{i=1}^{n} s^{\rho / r_{i}}\right)^{1 / \rho}$ and $\underline{\rho}_{r}^{-1}(s)=\left(\sum_{i=1}^{n} s^{\rho / r_{i}}\right)^{1 / \rho}$ as wanted from class $\mathcal{K}_{\infty}$.

Therefore, the proposed homogeneous norm is equivalent to the uniform norm in $C_{[a, b]}$. The homogeneous norm in the Banach space has the same important property that $\left\|\Lambda_{r}(\lambda) \phi\right\|_{r}=\lambda\|\phi\|_{r}$ for all $\phi \in C_{[a, b]}$. Define the corresponding unit sphere $\mathcal{S}_{r}=\left\{\phi \in C_{[-\tau, 0]}:\|\phi\|_{r}=1\right\}$.

Definition 1. The function $g: C_{[-\tau, 0]} \rightarrow \mathbb{R}$ is called $r$-homogeneous $\left(r_{i}>0\right.$, $i=\overline{1, n})$, if for any $\phi \in C_{[-\tau, 0]}$ the relation

$$
g\left(\Lambda_{r}(\lambda) \phi\right)=\lambda^{d} g(\phi)
$$

holds for some $d \in \mathbb{R}$ and all $\lambda>0$. 
The function $f: C_{[-\tau, 0]} \rightarrow \mathbb{R}^{n}$ is called $r$-homogeneous $\left(r_{i}>0, i=\overline{1, n}\right)$, if for any $\phi \in C_{[-\tau, 0]}$ the relation

$$
f\left(\Lambda_{r}(\lambda) \phi\right)=\lambda^{d} \Lambda_{r}(\lambda) f(\phi)
$$

holds for some $d \geq-\min _{1 \leq i \leq n} r_{i}$ and all $\lambda>0$.

In both cases, the constant $d$ is called the degree of homogeneity.

The introduced notion of homogeneity in $C_{[-\tau, 0]}$ is reduced to the standard one in $\mathbb{R}^{n}[6]$ under a vector argument substitution. An advantage of homogeneous systems described by nonlinear ordinary differential equations is that analysis of their stability can be performed on the unit sphere $\mathbb{S}_{r}$ only [6] (the homogeneous system trajectories have a similar behavior on any other sphere defined by the norm $\left.|\cdot|_{r}\right)$. This conclusion is based on the property that any solution of a homogeneous system can be obtained from another solution under the dilation rescaling and a suitable time re-parametrization. A similar property holds for some functional homogeneous systems.

Proposition 1. Let $x: \mathbb{R}_{+} \rightarrow \mathbb{R}^{n}$ be a solution of the r-homogeneous system (1) with the degree $d=0$ for an initial condition $x_{0} \in C_{[-\tau, 0]}$. For any $\lambda>0$ define $y(t)=\Lambda_{r}(\lambda) x\left(\lambda^{d} t\right)$ for all $t \geq 0$, then $y(t)$ is also a solution of (1) with the initial condition $y_{0}=\Lambda_{r}(\lambda) x_{0}$.

Proof. By definition $x_{\lambda^{d} t}(s)=x\left(\lambda^{d} t+\lambda^{d} s\right)=x(t+s) \in \mathbb{R}^{n}$ and $y_{t}(s)=\Lambda_{r}(\lambda) x_{\lambda^{d} t}(s)$ for any $-\tau \leq s \leq 0$, then

$$
\begin{aligned}
\dot{y}(t) & =\frac{d}{d t}\left(\Lambda_{r}(\lambda) x\left(\lambda^{d} t\right)\right)=\lambda^{d} \Lambda_{r}(\lambda) f\left(x_{\lambda^{d} t}\right) \\
& =f\left(\Lambda_{r}(\lambda) x_{\lambda^{d} t}\right)=f\left(y_{t}\right)
\end{aligned}
$$

and $y(t)$ is a solution of (1).

Corollary 1. Let the origin be locally asymptotically stable for a r-homogeneous system (1) with the degree $d=0$, then it is globally asymptotically stable.

Proof. Assume that the origin is locally attractive for (1) with an open domain of attraction $\mathcal{A} \subset C_{[-\tau, 0]}$, i.e. for any $\varepsilon>0$ and $x_{0} \in \mathcal{A}$ there is $T_{\varepsilon, x_{0}} \geq 0$ such that $\left\|x\left(t, x_{0}\right)\right\|_{r} \leq \varepsilon$ for all $t \geq T_{\varepsilon, x_{0}}$ (by Lemma 1 the norms $\|\cdot\|$ and $\|\cdot\|_{r}$ can be replaced). Take a $\mu>0$ such that $\mathcal{S}_{r}^{\mu} \subset \mathcal{A}$ where $\mathcal{S}_{r}^{\mu}=\mu \mathcal{S}_{r}$, then for any $\xi \in C_{[-\tau, 0]}$ there is $x_{0} \in \mathcal{S}_{r}^{\mu}$ such that $\xi=\Lambda_{r}(\lambda) x_{0}$ for $\lambda=\mu^{-1}\|\xi\|_{r}$ and the corresponding unique solution $x(t, \xi)=\Lambda_{r}(\lambda) x\left(\lambda^{d} t, x_{0}\right)$ by Proposition 1. Obviously, if $x\left(t, x_{0}\right) \rightarrow$ 0 for all $x_{0} \in \mathcal{S}_{r}^{\mu}$ with $t \rightarrow+\infty$, then so is $x(t, \xi)=\Lambda_{r}(\lambda) x\left(\lambda^{d} t, x_{0}\right)$, and the claims about global attractiveness and forward completeness follow.

To prove that local stability of the origin implies global in this case, assume that $\sup _{t \geq 0}\left\|x\left(t, x_{0}\right)\right\|_{r} \leq \sigma\left(\left\|x_{0}\right\|_{r}\right)$ for all $x_{0} \in \mathcal{A}$ and some $\sigma \in \mathcal{K}$. Now take any 
$\xi \in C_{[-\tau, 0]}$, then there is $x_{0} \in \mathcal{S}_{r}^{\mu} \subset \mathcal{A}$ such that $\xi=\Lambda_{r}(\lambda) x_{0}$ for $\lambda=\mu^{-1}\|\xi\|_{r}$ with the corresponding unique solution $x(t, \xi)=\Lambda_{r}(\lambda) x\left(\lambda^{d} t, x_{0}\right)$ by Proposition 1 . Therefore

$$
\begin{aligned}
\sup _{t \geq 0}\|x(t, \xi)\|_{r} & =\sup _{t \geq 0}\left\|\Lambda_{r}(\lambda) x\left(\lambda^{d} t, x_{0}\right)\right\|_{r}=\lambda \sup _{t \geq 0}\left\|x\left(\lambda^{d} t, x_{0}\right)\right\|_{r} \\
& \leq\|\xi\|_{r} \sigma\left(\left\|x_{0}\right\|_{r}\right) / \mu=\sigma(\mu) / \mu\|\xi\|_{r}
\end{aligned}
$$

and the system is Lyapunov stable [17, 18, 19].

Thus we have proven that for homogeneous time-delay systems with $d=0$ any local stability/attractivity properties at the origin hold globally. The case of homogeneous systems with $d=0$ becomes important for stability analysis using local homogeneous approximating dynamics, which are considered in Section 4 below (the approximating dynamics degree can be assigned to be zero).

For ordinary differential equations it has been also shown that asymptotically stable/unstable homogeneous systems always have homogeneous Lyapunov functions $[2,14,6]$. In this work we would like to prove a similar (sufficient only) result for time-delay homogeneous systems. For time-delay systems there exist two main techniques for stability analysis based on the Lyapunov approach. The first one is based on Lyapunov-Krasovskii functionals, another one on Lyapunov-Razumikhin functions [17, 18, 19]. Consider consequently both of them.

3.1. Lyapunov-Krasovskii approach. Unfortunately, due to peculiarities of the directional derivatives for functionals (see the definition in Section 2) the LyapunovKrasovskii approach is hard to develop using homogeneity for a general case. To explain the issue, consider the following example. Let the functions $f_{0}: \mathbb{R}^{n} \rightarrow \mathbb{R}^{n}$, $f_{1}: \mathbb{R}^{n} \rightarrow \mathbb{R}^{n}, g_{0}: \mathbb{R}^{n} \rightarrow \mathbb{R}_{+}, g_{1}: \mathbb{R}^{n} \rightarrow \mathbb{R}_{+}$be $r$-homogeneous with the same $r_{i}>$ $0, i=\overline{1, n}$ and similar degrees $d \geq-\min _{1 \leq i \leq n} r_{i}$ for $f_{0}, f_{1}$ and $v>\max \{0,-d\}$ for $g_{0}, g_{1}$. For $f\left(x_{t}\right)=f_{0}\left[x_{t}(0)\right]+f_{1}\left[x_{t}(-\tau)\right]$ in $(1)$ consider the Lyapunov-Krasovskii functional

$$
\mathcal{V}(\phi)=g_{0}[\phi(0)]+\int_{-\tau}^{0} g_{1}[\phi(s)] d s, \phi \in C_{[-\tau, 0]},
$$

which satisfies the required homogeneity conditions, then

$$
D^{+} \mathcal{V}(\phi)=\partial g_{0}(x) /\left.\partial x\right|_{x=\phi(0)}\left\{f_{0}[\phi(0)]+f_{1}[\phi(-\tau)]\right\}+g_{1}[\phi(0)]-g_{1}[\phi(-\tau)]
$$

Let for some $\varphi \in \mathcal{S}_{r}$ the above expression verify $\dot{\mathcal{V}}<0$. Consider the dilation transformation $\xi=\Lambda_{r}(\lambda) \varphi$ influence on $\dot{\mathcal{V}}$ :

$$
\begin{aligned}
D^{+} \mathcal{V}(\xi)=D^{+} \mathcal{V}\left(\Lambda_{r}(\lambda) \varphi\right)= & \lambda^{d+\nu} \partial g_{0}(x) /\left.\partial x\right|_{x=\varphi(0)}\left\{f_{0}[\varphi(0)]+\right. \\
& \left.f_{1}[\varphi(-\tau)]\right\}+\lambda^{\nu}\left\{g_{1}[\varphi(0)]-g_{1}[\varphi(-\tau)]\right\}
\end{aligned}
$$


Thus this basic transformation for homogeneous systems scales differently the parts proportional to $f_{0}, f_{1}$ and $g_{0}, g_{1}$, therefore in general case for some $\lambda>0$ it could be $\dot{\mathcal{V}}>0$. In other words, the derivative of a homogeneous functional may be non-homogeneous. As we have shown, the only exclusion is the case with $d=0$ (that is the case of linear time-delay systems, for example, but not only).

Proposition 2. Let in (1) the function $f\left(x_{t}\right)=f_{0}\left[x_{t}(0)\right]+f_{1}\left[x_{t}(-\tau)\right]$ have $r$ homogeneous $f_{0}: \mathbb{R}^{n} \rightarrow \mathbb{R}^{n}$ and $f_{1}: \mathbb{R}^{n} \rightarrow \mathbb{R}^{n}$ with degree $d=0$, and there exist $r$-homogeneous continuous functions $g_{0}: \mathbb{R}^{n} \rightarrow \mathbb{R}_{+}, g_{1}: \mathbb{R}^{n} \rightarrow \mathbb{R}_{+}$with degree $\nu>0$ such that $D^{+} \mathcal{V}(\varphi)<0$ for all $\varphi \in \mathcal{S}_{r}$, where $\mathcal{V}$ is given in (2). Then $D^{+} \mathcal{V}(\phi)<0$ for all $\phi \in C_{[-\tau, 0]}$ with $\|\phi\| \neq 0$.

A similar result can be proven for a non-homogeneous functional $\mathcal{V}$ with a homogeneous derivative $D^{+} \mathcal{V}(\varphi)$, i.e. if the function $g_{0}$ is $r$-homogeneous with degree $\mu$, the function $g_{1}$ is $r$-homogeneous with degree $\nu$ and the functions $f_{0}, f_{1}$ are $r$-homogeneous with degree $d$, provided that $\mu+d=\nu$.

3.2. Lyapunov-Razumikhin approach. The Razumikhin approach is based on Lyapunov-Razumikhin functions $[17,18,19]$ defined on $\mathbb{R}^{n}$, which give a pointwise sufficient criteria for stability (not a functional one). It allows us to develop this approach using homogeneous arguments.

Note, that if a function $V: \mathbb{R}^{n} \rightarrow \mathbb{R}_{+}$is positive definite and radially unbounded, then there are functions $\alpha_{1}, \alpha_{2} \in \mathcal{K}_{\infty}$ such that $\alpha_{1}(|x|) \leq V(x) \leq \alpha_{2}(|x|)$ for all $x \in \mathbb{R}^{n}$. If $V$ is $r$-homogeneous with a degree $\nu$, then for any $x \in \mathbb{R}^{n}$ we have $V(x)=V\left(\Lambda_{r}\left(|x|_{r}\right) y\right)=|x|_{r}^{\nu} V(y)$ for some $y \in \mathbb{S}_{r}$, therefore, in this case $\alpha_{1}(s)=\left[\bar{\sigma}_{r}^{-1}(s)\right]^{\nu} \min _{y \in S_{r}} V(y)$ and $\alpha_{2}(s)=\left[\underline{\sigma}_{r}^{-1}(s)\right]^{\nu} \max _{y \in S_{r}} V(y)$, and $\nu$ should be strictly positive for radial unboundedness of $V$. In addition, if $V$ is continuously differentiable at the origin, then $\nu$ should be bigger than 1 .

Theorem 1. Let the function $f$ in (1) be r-homogeneous with degree $d \geq-\min _{1 \leq i \leq n} r_{i}$ and there exist a locally Lipschitz continuous $r$-homogeneous Lyapunov-Razumikhin function $V: \mathbb{R}^{n} \rightarrow \mathbb{R}_{+}$with degree $\nu>\max \{0,-d\}$ such that

(i) there exist functions $\alpha, \gamma \in \mathcal{K}$ such that for all $\varphi \in \mathcal{S}_{r}$

$$
\max _{\theta \in[-\tau, 0]} V[\varphi(\theta)]<\gamma\{V[\varphi(0)]\} \Rightarrow D^{+} V[\varphi(0)] f(\varphi) \leq-\alpha(|\varphi(0)|) ;
$$

(ii) there exists a function $\gamma^{\prime} \in \mathcal{K}$ such that $\lambda s<\gamma^{\prime}(\lambda s) \leq \lambda \gamma(s)$ for all $s, \lambda \in$ $\mathbb{R}_{+} \backslash\{0\}$.

Then the origin is globally asymptotically stable for the system (1).

Proof. The dilation transformation $\phi=\Lambda_{r}(\lambda) \varphi$ connects any $\phi \in C_{[-\tau, 0]} \backslash\{0\}$ with some $\varphi \in \mathcal{S}_{r}$ for properly chosen $\lambda>0$. Let us multiply the left-hand part of the 
implication (i) by $\lambda^{\nu}$ and the right-hand part by $\lambda^{\nu+d}$ :

$$
\max _{\theta \in[-\tau, 0]} \lambda^{\nu} V[\varphi(\theta)]<\lambda^{\nu} \gamma\{V[\varphi(0)]\} \Rightarrow \lambda^{\nu+d} D^{+} V[\varphi(0)] f(\varphi) \leq-\lambda^{\nu+d} \alpha(|\varphi(0)|),
$$

the inequalities and relations should not be changed for $\lambda>0$. Owing the functions $\gamma$ and $\gamma^{\prime}$ properties, the inequality $\gamma^{\prime}\left\{\lambda^{\nu} V[\varphi(0)]\right\} \leq \lambda^{\nu} \gamma\{V[\varphi(0)]\}$ holds. Formally $\inf _{\varphi \in \mathcal{S}_{r}}\{\alpha(|\varphi(0)|)\}=0$, however having in mind that $\varphi$ is a continuous function and a solution of (1), there exists

$$
a=\inf _{\varphi \in \mathcal{S}_{r}, \max _{\theta \in[-\tau, 0]} V[\varphi(\theta)]<\gamma\{V[\varphi(0)]\}} \alpha(|\varphi(0)|)
$$

and $a>0$. Indeed, for the case $\max _{\theta \in[-\tau, 0]} V[\varphi(\theta)]<\gamma\{V[\varphi(0)]\}$ we have

$$
\begin{aligned}
\alpha_{1}\left(\underline{\rho}_{r}(1)\right) & \leq \alpha_{1}(|| \varphi||)=\alpha_{1}\left(\max _{\theta \in[-\tau, 0]}|\varphi(\theta)|\right) \\
& =\max _{\theta \in[-\tau, 0]} \alpha_{1}(|\varphi(\theta)|) \leq \max _{\theta \in[-\tau, 0]} V(\varphi(\theta)) \\
& <\gamma[V(\varphi(0))],
\end{aligned}
$$

thus $\alpha_{2}^{-1} \circ \gamma^{-1} \circ \alpha_{1}\left(\underline{\rho}_{r}(1)\right)<|\varphi(0)|$ for all such $\varphi \in \mathcal{S}_{r}$ and $a \geq \alpha_{2}^{-1} \circ \gamma^{-1} \circ \alpha_{1}\left(\underline{\rho}_{r}(1)\right)>$ 0 . Therefore, due to homogeneity of the functions $f$ and $V$ we have:

$$
\begin{gathered}
\max _{\theta \in[-\tau, 0]} V\left[\Lambda_{r}(\lambda) \varphi(\theta)\right]<\gamma^{\prime}\left\{V\left[\Lambda_{r}(\lambda) \varphi(0)\right]\right\} \Rightarrow \\
D^{+} V\left[\Lambda_{r}(\lambda) \varphi(0)\right] f\left(\Lambda_{r}(\lambda) \varphi\right) \leq-\lambda^{\nu+d} a,
\end{gathered}
$$

or equivalently $\left(\lambda=|| \phi \|_{r} \geq|\phi(0)|_{r} \geq \bar{\sigma}_{r}^{-1}(|\phi(0)|)\right)$,

$$
\max _{\theta \in[-\tau, 0]} V[\phi(\theta)]<\gamma^{\prime}\{V[\phi(0)]\} \Rightarrow D^{+} V[\phi(0)] f(\phi) \leq-\alpha^{\prime}(|\phi(0)|),
$$

where the function $\alpha^{\prime}(s)=\left(\bar{\sigma}_{r}^{-1}(s)\right)^{\nu+d} a$ is from class $\mathcal{K}$ since $\nu+d>0$. Therefore, if Razumikhin arguments are true for $\varphi \in \mathcal{S}_{r}$, then they are valid for any $\phi \in C_{[-\tau, 0]}$, that implies the global asymptotic stability of the origin for $(1)[17,18,19]$.

The condition (i) imposed in Theorem 1 on the system (1) behavior is the conventional Razumikhin condition (except that in the homogeneous case it can be verified on the sphere $\mathcal{S}_{r}$ only). The constraint (ii) on existence of the function $\gamma^{\prime}$ is new. Roughly speaking this requirement says that the function $\gamma$ has to be "homogeneous" with degree 1. Another explanation is that the function $\gamma$ is globally Lipschitz. For instance, this is the case if there exists $1<k_{1}<k_{2}<+\infty$ such that $k_{1} s \leq \gamma(s) \leq k_{2} s$

Remark 1. Note that the full derivative $D^{+} V[\varphi(0)] f(\varphi)$ is a function of $\varphi \in \mathcal{S}_{r}$, however under the condition $\max _{\theta \in[-\tau, 0]} V\left[\Lambda_{r}(\lambda) \varphi(\theta)\right]<\gamma^{\prime}\left\{V\left[\Lambda_{r}(\lambda) \varphi(0)\right]\right\}$ the inequality $D^{+} V[\varphi(0)] f(\varphi)<m(\varphi(0))$ holds for some $m: \mathbb{R}^{n} \rightarrow \mathbb{R}$. If the function $m$ 
is $r$-homogeneous (the functions $V$ and $f$ possess this property), then the property $m(\varphi(0)) \leq-\alpha(|\varphi(0)|)$ in (ii) has to be verified for $\varphi(0) \in \mathbb{S}_{r}$ only.

Instability conditions in the Lyapunov-Razumikhin framework have been formulated in $[31,32]$. We will say that the system (1) is unstable at the origin if for any $\delta>0$ there exist $\varepsilon>0,\left\|x_{0}\right\| \leq \delta$ and $t_{x_{0}, \varepsilon}^{\prime} \geq 0$ such that $\left\|x_{t_{x_{0}, \varepsilon}^{\prime}}\right\|>\varepsilon$. For a function $V: \mathbb{R}^{n} \rightarrow \mathbb{R}_{+}$define two sets:

$$
\begin{aligned}
& P_{M}^{V}=\left\{\varphi \in \mathcal{S}_{r}: V[\varphi(0)]=\max _{\theta \in[-\tau, 0]} V[\varphi(\theta)]\right\}, \\
& P_{m}^{V}=\left\{\varphi \in \mathcal{S}_{r}: V[\varphi(0)]=\min _{\theta \in[-\tau, 0]} V[\varphi(\theta)]\right\} .
\end{aligned}
$$

Theorem 2. Let the function $f$ in (1) be r-homogeneous with degree $d \geq-\min _{1 \leq i \leq n} r_{i}$ and there exist a locally Lipschitz continuous r-homogeneous Lyapunov-Razumikhin function $V: \mathbb{R}^{n} \rightarrow \mathbb{R}_{+}$with degree $\nu>\max \{0,-d\}$ such that one of the following properties is satisfied:

(i) $D^{-} V[\varphi(0)] f(\varphi)>0$ for all $\varphi \in P_{M}^{V}$;

(ii) $D^{-} V[\varphi(0)] f(\varphi)>0$ for all $\varphi \in P_{m}^{V}$.

Then the system (1) is unstable at the origin.

Proof. Select a $\lambda>0$ such that the dilation transformation $\phi=\Lambda_{r}(\lambda) \varphi$ connects a $\phi \in C_{[-\tau, 0]} \backslash\{0\}$ with some $\varphi \in \mathcal{S}_{r}$. The sets $\Pi_{M}^{V}=\left\{\phi \in C_{[-\tau, 0]}: V[\phi(0)]=\right.$ $\left.\max _{\theta \in[-\tau, 0]} V[\phi(\theta)]\right\}, \Pi_{m}^{V}=\left\{\phi \in C_{[-\tau, 0]}: V[\phi(0)]=\min _{\theta \in[-\tau, 0]} V[\phi(\theta)]\right\}$ are well defined by the dilation transformation of $P_{M}^{V}, P_{m}^{V}$ :

$$
\begin{aligned}
\cup_{\lambda>0} \Lambda_{r}(\lambda) P_{M}^{V} & =\left\{\phi=\Lambda_{r}(\lambda) \varphi, \lambda>0, \varphi \in \mathcal{S}_{r}: V[\varphi(0)]=\max _{\theta \in[-\tau, 0]} V[\varphi(\theta)]\right\} \\
& =\left\{\phi=\Lambda_{r}(\lambda) \varphi, \lambda>0, \varphi \in \mathcal{S}_{r}: \lambda^{\nu} V[\varphi(0)]=\lambda^{\nu} \max _{\theta \in[-\tau, 0]} V[\varphi(\theta)]\right\} \\
& =\Pi_{M}^{V}, \\
\cup_{\lambda>0} \Lambda_{r}(\lambda) P_{m}^{V} & =\left\{\phi=\Lambda_{r}(\lambda) \varphi, \lambda>0, \varphi \in \mathcal{S}_{r}: V[\varphi(0)]=\min _{\theta \in[-\tau, 0]} V[\varphi(\theta)]\right\} \\
& =\left\{\phi=\Lambda_{r}(\lambda) \varphi, \lambda>0, \varphi \in \mathcal{S}_{r}: \lambda^{\nu} V[\varphi(0)]=\lambda^{\nu} \min _{\theta \in[-\tau, 0]} V[\varphi(\theta)]\right\} \\
& =\Pi_{m}^{V} .
\end{aligned}
$$

Take $\phi \in \Pi_{M}^{V}$ or $\phi \in \Pi_{m}^{V}$, then

$$
D^{-} V[\phi(0)] f(\phi)=\lambda^{\nu+d} D^{-} V[\varphi(0)] f(\varphi)>0 .
$$

Therefore, $D^{-} V[\phi(0)] f(\phi)>0$ for all $\phi \in \Pi_{M}^{V}$ or $\phi \in \Pi_{m}^{V}$, which are the conditions of instability of (1) into the sets $\Pi_{M}^{V}$ or $\Pi_{m}^{V}$ from [31,32].

In the case (i) of Theorem 2, the set $\Pi_{M}^{V}$ is forward invariant for the system (1) (once the relation $V[\phi(0)]=\max _{\theta \in[-\tau, 0]} V[\phi(\theta)]$ is valid, next it will be satisfied for all forward times since $\dot{V}(\phi)>0$ on $\Pi_{M}^{V}$ ), thus all trajectories initiated or entered 
$\Pi_{M}^{V}$ go to infinity staying into $\Pi_{M}^{V}$. The case (ii) is more tricky, then we have $C_{[-\tau, 0]}=\Pi_{m}^{V} \cup \Theta_{m}^{V}$, where $\Theta_{m}^{V}=\left\{\phi \in C_{[-\tau, 0]}: V[\phi(0)]>\min _{\theta \in[-\tau, 0]} V[\phi(\theta)]\right\}$. The function $V(t)=V(x(t))$ is strictly increasing in $\Pi_{m}^{V}$ (since $\dot{V}(\phi)>0$ on $\Pi_{m}^{V}$ ) and a trajectory of (1) cannot stay continuously in $\Pi_{m}^{V}$ on a time interval of the length more than $\tau$ (by the same reason: $V(t)$ is strictly increasing while $x(t)$ is in $\Pi_{m}^{V}$ ). In $\Theta_{m}^{V}$, by the definition of this set, the function $V_{m}(t)=\min _{\theta \in[-\tau, 0]} V[x(t+\theta)]$, which is defined on trajectories of (1), is non-decreasing while a trajectory stays in $\Theta_{m}^{V}$ (since $V[\phi(0)]>\min _{\theta \in[-\tau, 0]} V[\phi(\theta)]$ if $\phi \in \Theta_{m}^{V}$ ), and if a trajectory belongs to $\Theta_{m}^{V}$ a time bigger than $\tau$ we have that $V_{m}(t)>V_{m}(t-\tau)$. Since a trajectory by leaving $\Theta_{m}^{V}$ enters the set $\Pi_{m}^{V}$, where the function $V(t)$ is strictly increasing and $V_{m}(t)=V(t)$, it implies that the property $V_{m}(t)>V_{m}(t-\tau)$ is satisfied for all $t \geq 0$. Therefore, all trajectories in the case (ii) go to infinity. The case (i) may include the case of a saddle equilibrium existence for (1), while the case (ii) corresponds to anti-stable or strongly unstable equilibrium of (1) at the origin.

The results of theorems 1 and 2 mean that by using homogeneous LyapunovRazumikhin functions the global stability/instability of a homogeneous system at the origin can be checked on the sphere $\mathcal{S}_{r}$ only. These facts may simplify the function $V$ search and the system analysis with application of a numerical routine. The drawback is that in the space $C_{[-\tau, 0]}$, the sphere $\mathcal{S}_{r}$ is a rather complex object.

\section{LOCAL HOMOGENEITY}

A disadvantage of the global homogeneity introduced so far is that such systems possess the same behavior "globally". Thus the homogeneous systems are not really "nonlinear", they have similar diversity of operating modes as linear systems. In fact, from analysis and design points of view the homogeneous systems are a generalization of linear ones. Comparing with other nonlinear systems, it may be easier to find a (homogeneous) Lyapunov function for homogeneous systems. That is why finding a possibility to apply this approach for a broader class of nonlinear systems is very important.

An approach to resolve this issue consists in introducing a local version of homogeneity as in [14].

Definition 2. The function $g: C_{[-\tau, 0]} \rightarrow \mathbb{R}$ is called $\left(r, \lambda_{0}, g_{0}\right)$-homogeneous $\left(r_{i}>\right.$ $\left.0, i=\overline{1, n} ; g_{0}: C_{[-\tau, 0]} \rightarrow \mathbb{R}\right)$ if for any $\phi \in \mathcal{S}_{r}$ the relation

$$
\lim _{\lambda \rightarrow \lambda_{0}} \lambda^{-d_{0}} g\left(\Lambda_{r}(\lambda) \phi\right)-g_{0}(\phi)=0
$$

is satisfied (uniformly on $\mathcal{S}_{r}$ for $\lambda_{0} \in\{0,+\infty\}$ ) for some $d_{0} \in \mathbb{R}$. 
The system (1) is called $\left(r, \lambda_{0}, f_{0}\right)$-homogeneous $\left(r_{i}>0, i=\overline{1, n} ; f_{0}: C_{[-\tau, 0]} \rightarrow\right.$ $\left.\mathbb{R}^{n}\right)$ if for any $\phi \in \mathcal{S}_{r}$ the relation

$$
\lim _{\lambda \rightarrow \lambda_{0}} \lambda^{-d_{0}} \Lambda_{r}^{-1}(\lambda) f\left(\Lambda_{r}(\lambda) \phi\right)-f_{0}(\phi)=0
$$

is satisfied (uniformly on $\mathcal{S}_{r}$ for $\lambda_{0} \in\{0,+\infty\}$ ) for some $d_{0} \geq-\min _{1 \leq i \leq n} r_{i}$.

For a given $\lambda_{0}, g_{0}$ and $f_{0}$ are called approximating functions.

For any $0<\lambda_{0}<+\infty$ the following formulas give a variant of homogeneous approximating functions $g_{0}$ and $f_{0}$ :

$$
\begin{aligned}
& g_{0}(\phi)=\|\phi\|_{r}^{d} \lambda_{0}^{-d_{0}} g\left(\Lambda_{r}\left(\lambda_{0}\right) \Lambda_{r}^{-1}\left(\|\phi\|_{r}\right) \phi\right), d \geq 0, \\
& f_{0}(\phi)=\|\phi\|_{r}^{d} \lambda_{0}^{-d_{0}} \Lambda_{r}\left(\|\phi\|_{r}\right) \Lambda_{r}^{-1}\left(\lambda_{0}\right) f\left(\Lambda_{r}\left(\lambda_{0}\right) \Lambda_{r}^{-1}\left(\|\phi\|_{r}\right) \phi\right), d \geq-\min _{1 \leq i \leq n} r_{i} .
\end{aligned}
$$

This property is called local homogeneity [14], it allows us to analyze local stability/instability of the system (1) on the basis of a simplified system

$$
d y(t) / d t=f_{0}\left[y_{\tau}(t)\right], t \geq 0,
$$

called the local approximating dynamics for (1).

Theorem 3. Let the system (1) be $\left(r, \lambda_{0}, f_{0}\right)$-homogeneous for some $r_{i}>0, i=\overline{1, n}$, the function $f_{0}$ be continuous and $r$-homogeneous with the degree $d_{0}$. Suppose there exists a locally Lipschitz continuous $r$-homogeneous Lyapunov-Razumikhin function $V_{0}: \mathbb{R}^{n} \rightarrow \mathbb{R}_{+}$with the degree $\nu_{0}>\max \left\{0,-d_{0}\right\}, \alpha_{1}(|x|) \leq V_{0}(x) \leq \alpha_{2}(|x|)$ for all $x \in \mathbb{R}^{n}$ and some $\alpha_{1}, \alpha_{2} \in \mathcal{K}_{\infty}$ such that:

(i) there exist functions $\alpha, \gamma \in \mathcal{K}$ such that for all $\varphi \in \mathcal{S}_{r}$

$$
\max _{\theta \in[-\tau, 0]} V_{0}[\varphi(\theta)]<\gamma\left\{V_{0}[\varphi(0)]\right\} \Rightarrow D^{+} V_{0}[\varphi(0)] f_{0}(\varphi) \leq-\alpha(|\varphi(0)|) ;
$$

(ii) there exists a function $\gamma^{\prime} \in \mathcal{K}$ such that $\lambda s<\gamma^{\prime}(\lambda s) \leq \lambda \gamma(s)$ for all $s, \lambda \in$ $\mathbb{R}_{+} \backslash\{0\}$.

Then

1) if $\lambda_{0}=0$, then there exists $0<\bar{\lambda}_{\varepsilon}$ such that the system (1) is locally asymptotically stable at the origin with the domain of attraction containing the set

$$
X_{0}=\left\{\phi \in C_{[-\tau, 0]}:\|\phi\| \leq \alpha_{1}^{-1} \circ \alpha_{2} \circ \bar{\rho}_{r}\left(\bar{\lambda}_{\varepsilon}\right)\right\} ;
$$

2) if $\lambda_{0}=+\infty$, then there exists $0<\underline{\lambda}_{\varepsilon}<+\infty$ such that the system (1) is globally asymptotically stable with respect to forward invariant set

$$
X_{\infty}=\left\{\phi \in C_{[-\tau, 0]}:\|\phi\| \leq \alpha_{1}^{-1} \circ \alpha_{2} \circ \underline{\rho}_{r}\left(\underline{\lambda}_{\varepsilon}\right)\right\} ;
$$

3) if $0<\lambda_{0}<+\infty$, then there exist $0<\underline{\lambda}_{\varepsilon} \leq \lambda_{0} \leq \bar{\lambda}_{\varepsilon}<+\infty$ such that the system (1) is asymptotically stable with respect to the forward invariant set $X_{\infty}$ with region 
of attraction

$$
\begin{aligned}
X= & \left\{\phi \in C_{[-\tau, 0]}: \alpha_{1}^{-1} \circ \alpha_{2} \circ \underline{\rho}_{r}\left(\underline{\lambda}_{\varepsilon}\right)<\|\phi\|\right. \\
& \left.<\alpha_{1}^{-1} \circ \alpha_{2} \circ \bar{\rho}_{r}\left(\bar{\lambda}_{\varepsilon}\right)\right\}
\end{aligned}
$$

provided that the set $X$ is connected and nonempty.

Proof. For the system (1) the transformation of coordinates $\phi=\Lambda_{r}(\lambda) \varphi$ connects any $\phi \in C_{[-\tau, 0]} \backslash\{0\}$ with some $\varphi \in \mathcal{S}_{r}$ for $\lambda=\|\phi\|_{r}$. According to definition of the function $V_{0}$ we have:

$$
\begin{aligned}
D^{+} V_{0}[\phi(0)] f(\phi)= & D^{+} V_{0}\left[\Lambda_{r}(\lambda) \varphi(0)\right] f\left(\Lambda_{r}(\lambda) \varphi\right) \\
= & D^{+} V_{0}\left[\Lambda_{r}(\lambda) \varphi(0)\right]\left\{f_{0}\left(\Lambda_{r}(\lambda) \varphi\right)+\left[f\left(\Lambda_{r}(\lambda) \varphi\right)-f_{0}\left(\Lambda_{r}(\lambda) \varphi\right)\right]\right\} \\
= & \lambda^{d_{0}+\nu_{0}} D^{+} V_{0}[\varphi(0)] f_{0}(\varphi) \\
& +\lambda^{\nu_{0}} D^{+} V_{0}[\varphi(0)] \Lambda_{r}^{-1}(\lambda)\left\{f\left(\Lambda_{r} \varphi\right)-\lambda^{d_{0}} \Lambda_{r}(\lambda) f_{0}(\varphi)\right\} \\
= & \lambda^{d_{0}+\nu_{0}} D^{+} V_{0}[\varphi(0)]\left\{f_{0}(\varphi)+\left[\lambda^{-d_{0}} \Lambda_{r}^{-1}(\lambda) f\left(\Lambda_{r}(\lambda) \varphi\right)-f_{0}(\varphi)\right]\right\} .
\end{aligned}
$$

Due to continuity of the functions $f, f_{0}$ and the local homogeneity property definition for any $\varepsilon>0$ there exist $\underline{\lambda}_{\varepsilon} \leq \lambda_{0} \leq \bar{\lambda}_{\varepsilon}$ such that

$$
\sup _{\varphi \in \mathcal{S}_{r}}\left|D^{+} V_{0}[\varphi(0)]\left\{\lambda^{-d_{0}} \Lambda_{r}^{-1}(\lambda) f\left(\Lambda_{r}(\lambda) \varphi\right)-f_{0}(\varphi)\right\}\right| \leq \varepsilon
$$

for all $\lambda \in\left(\underline{\lambda}_{\varepsilon}, \bar{\lambda}_{\varepsilon}\right)$. From (i) we know that the inequality $D^{+} V_{0}[\varphi(0)] f_{0}(\varphi) \leq$ $-\alpha(|\varphi(0)|)$ is satisfied under the condition $\max _{\theta \in[-\tau, 0]} V_{0}[\varphi(\theta)]<\gamma\left\{V_{0}[\varphi(0)]\right\}$. By the same arguments, since the property $\max _{\theta \in[-\tau, 0]} V_{0}[\varphi(\theta)]<\gamma\left\{V_{0}[\varphi(0)]\right\}$ excludes from consideration some functions $\varphi \in \mathcal{S}_{r}$, we can prove that $a>0$, where

$$
a=\inf _{\varphi \in \mathcal{S}_{r}, \max _{\theta \in[-\tau, 0]} V_{0}[\varphi(\theta)]<\gamma\left\{V_{0}[\varphi(0)]\right\}} \alpha(|\varphi(0)|) .
$$

Let the constants $\underline{\lambda}_{\varepsilon}, \bar{\lambda}_{\varepsilon}$ be chosen to ensure that $a>\varepsilon$. Multiplying (i) on $\lambda^{\nu_{0}}$ we get that if a property is satisfied for $\varphi \in \mathcal{S}_{r}$ with $\max _{\theta \in[-\tau, 0]} V_{0}[\varphi(\theta)]<\gamma\left\{V_{0}[\varphi(0)]\right\}$, then it also holds for $\phi \in C_{[-\tau, 0]}$ under the restriction $\max _{\theta \in[-\tau, 0]} V_{0}[\phi(\theta)]<$ $\gamma^{\prime}\left\{V_{0}[\phi(0)]\right\}$ (due to condition (ii)). Then the following property holds for $\alpha^{\prime}(s)=$ $\left(\bar{\sigma}_{r}^{-1}(s)\right)^{\nu_{0}+d_{0}}(a-\varepsilon)$ :

$$
\max _{\theta \in[-\tau, 0]} V_{0}[\phi(\theta)]<\gamma^{\prime}\left\{V_{0}[\phi(0)]\right\} \Rightarrow D^{+} V_{0}[\phi(0)] f_{0}(\phi) \leq-\alpha^{\prime}(|\phi(0)|),
$$

where $\phi=\Lambda_{r}(\lambda) \varphi, \varphi \in \mathcal{S}_{r}$ and $\lambda \in\left(\underline{\lambda}_{\varepsilon}, \bar{\lambda}_{\varepsilon}\right)$, i.e. for all $\phi \in X_{r}=\left\{\phi \in C_{[-\tau, 0]}\right.$ : $\left.\underline{\lambda}_{\varepsilon}<\|\phi\|_{r}<\bar{\lambda}_{\varepsilon}\right\}$.

If $\lambda_{0}=0$, then clearly $\underline{\lambda}_{\varepsilon}=0$ and the origin is locally asymptotically stable with the domain of asymptotic stability containing the set $X_{0}[17,19]$. Indeed, take any initial conditions $\|\phi\|_{r}<\bar{\lambda}_{\varepsilon}$, then $\max _{\theta \in[-\tau, 0]} V_{0}[\phi(\theta)] \leq \alpha_{2}\left(\bar{\rho}_{r}\left(\bar{\lambda}_{\varepsilon}\right)\right)$ and $V_{0}(t)$ is 
not increasing for all $t \geq 0$, therefore $|x(t)| \leq \alpha_{1}^{-1} \circ \alpha_{2} \circ \bar{\rho}_{r}\left(\bar{\lambda}_{\varepsilon}\right)$ in (1) for all $t \geq 0$, which gives the required conclusion.

If $\lambda_{0}=+\infty$, then $\bar{\lambda}_{\varepsilon}=+\infty$ and the function $V_{0}$ for the system (1) is decreasing into the set $\mathbb{R}^{n} \backslash X_{\infty}$ for some $\underline{\lambda}_{\varepsilon}$. Thus the set $X_{\infty}$ is forward invariant for (1) and attracting. Borrowing arguments from [17, 18] these facts imply the global asymptotic stability of the system (1) with respect to the set $X_{\infty}$.

Finally, let $0<\lambda_{0}<+\infty$ and the set $X$ be nonempty and connected, since $0 \leq|\phi(0)|<0.5 \alpha_{1}^{-1} \circ \alpha_{2} \circ \bar{\rho}_{r}\left(\bar{\lambda}_{\varepsilon}\right)$ for all $\phi \in X$, then the set $X$ contains a level of the function $V_{0}$ and the function $V_{0}$ is decreasing into the set $X$ and all trajectories $x\left(t, x_{0}\right)$ with initial conditions $x_{0} \in X$ reach for the set $X_{\infty}$.

Theorem 4. Let the system (1) be ( $\left.r, \lambda_{0}, f_{0}\right)$-homogeneous for some $r_{i}>0, i=\overline{1, n}$, the function $f_{0}$ be continuous and $r$-homogeneous with the degree $d_{0}$. Suppose there exists a locally Lipschitz continuous r-homogeneous Lyapunov-Razumikhin function $V_{0}: \mathbb{R}^{n} \rightarrow \mathbb{R}_{+}$with the degree $\nu_{0}>\max \left\{0,-d_{0}\right\}, \alpha_{1}(|x|) \leq V_{0}(x) \leq \alpha_{2}(|x|)$ for all $x \in \mathbb{R}^{n}$ and some $\alpha_{1}, \alpha_{2} \in \mathcal{K}_{\infty}$, such that $D^{-} V_{0}[\varphi(0)] f_{0}(\varphi) \geq a>0$ for all $\varphi \in P_{m}^{V_{0}}$ or $\varphi \in P_{M}^{V_{0}}$. Then

1) if $\lambda_{0}=0$, then there exists $0<\bar{\lambda}_{\varepsilon}$ such that for the system (1) the set

$$
X_{0}=\left\{\phi \in C_{[-\tau, 0]}:\|\phi\| \leq \alpha_{1}^{-1} \circ \alpha_{2} \circ \bar{\rho}_{r}\left(\bar{\lambda}_{\varepsilon}\right)\right\}
$$

is unstable;

2) if $\lambda_{0}=+\infty$, then there exists $0<\underline{\lambda}_{\varepsilon}<+\infty$ such that for the system (1) the set

$$
X_{\infty}=\left\{\phi \in C_{[-\tau, 0]}:\|\phi\| \leq \alpha_{1}^{-1} \circ \alpha_{2} \circ \underline{\rho}_{r}\left(\underline{\lambda}_{\varepsilon}\right)\right\}
$$

is unstable;

3) if $0<\lambda_{0}<+\infty$, then there exist $0<\underline{\lambda}_{\varepsilon} \leq \lambda_{0} \leq \bar{\lambda}_{\varepsilon}<+\infty$ such that for the system (1) the set $X_{\infty}$ is unstable provided that the set $X=\left\{\phi \in C_{[-\tau, 0]}\right.$ : $\left.\alpha_{1}^{-1} \circ \alpha_{2} \circ \underline{\rho}_{r}\left(\underline{\lambda}_{\varepsilon}\right)<\|\phi\|<\alpha_{1}^{-1} \circ \alpha_{2} \circ \bar{\rho}_{r}\left(\bar{\lambda}_{\varepsilon}\right)\right\}$ is connected and non empty.

Proof. For $\lambda=\|\phi\|_{r}$ the coordinates transformation $\phi=\Lambda_{r}(\lambda) \varphi$ connects any $\phi \in C_{[-\tau, 0]} \backslash\{0\}$ with some $\varphi \in \mathcal{S}_{r}$. As in the proof of Theorem 3 we have:

$D^{-} V_{0}[\phi(0)] f(\phi)=\lambda^{d_{0}+\nu_{0}} D^{-} V_{0}[\varphi(0)]\left\{f_{0}(\varphi)+\left[\lambda^{-d_{0}} \Lambda_{r}^{-1}(\lambda) f\left(\Lambda_{r}(\lambda) \varphi\right)-f_{0}(\varphi)\right]\right\}$.

Due to continuity of the functions $f, f_{0}$ and the local homogeneity property definition for any $\varepsilon>0$ there exist $\underline{\lambda}_{\varepsilon} \leq \lambda_{0} \leq \bar{\lambda}_{\varepsilon}$ such that

$$
\sup _{\varphi \in \mathcal{S}_{r}}\left|D^{-} V_{0}[\varphi(0)]\left\{\lambda^{-d_{0}} \Lambda_{r}^{-1}(\lambda) f\left(\Lambda_{r}(\lambda) \varphi\right)-f_{0}(\varphi)\right\}\right| \leq \varepsilon
$$

for all $\lambda \in\left(\underline{\lambda}_{\varepsilon}, \bar{\lambda}_{\varepsilon}\right)$. Let the constants $\underline{\lambda}_{\varepsilon}, \bar{\lambda}_{\varepsilon}$ be chosen to ensure that $a>\varepsilon$. As it was shown in the proof of Theorem $2, \Pi_{m}^{V_{0}}=\cup_{\lambda>0} \Lambda_{r}(\lambda) P_{m}^{V_{0}}$ and $\Pi_{M}^{V_{0}}=$ $\cup_{\lambda>0} \Lambda_{r}(\lambda) P_{M}^{V_{0}}$. Define $\Omega_{\varepsilon}=\left\{\phi \in C_{[-\tau, 0]}: \underline{\lambda}_{\varepsilon}<\|\phi\|_{r}<\bar{\lambda}_{\varepsilon}\right\}$, then the set $\Pi_{M}^{V_{0}} \cap \Omega_{\varepsilon}$ 
contains repelling trajectories of the system (1) $\left(D^{-} V_{0}[\phi(0)] f(\phi) \geq\|\phi\|_{r}^{\nu_{0}+d_{0}}(a-\right.$ $\varepsilon)>0$ for all trajectories in $\left.\Pi_{M}^{V_{0}} \cap \Omega_{\varepsilon}\right)$. Recall that $C_{[-\tau, 0]}=\Pi_{m}^{V_{0}} \cup \Theta_{m}^{V_{0}}$, where $\Theta_{m}^{V_{0}}=$ $\left\{\phi \in C_{[-\tau, 0]}: V_{0}[\phi(0)]>\min _{\theta \in[-\tau, 0]} V_{0}[\phi(\theta)]\right\}$, then $\Omega_{\varepsilon}=\left[\Pi_{m}^{V_{0}} \cap \Omega_{\varepsilon}\right] \cup\left[\Theta_{m}^{V_{0}} \cap \Omega_{\varepsilon}\right]$. According to performed computations, $D^{-} V_{0}[\phi(0)] f(\phi) \geq\|\phi\|_{r}^{\nu_{0}+d_{0}}(a-\varepsilon)>0$ for all $\phi \in \Pi_{m}^{V_{0}} \cap \Omega_{\varepsilon}$, therefore, the function $V_{0}$ is strictly increasing on trajectories of (1) into the set $\Pi_{m}^{V_{0}} \cap \Omega_{\varepsilon}$. In addition, $\dot{V}_{m}^{0}(t)=\dot{V}_{0}(t)$ for (1) for all trajectories in $\Pi_{m}^{V_{0}} \cap \Omega_{\varepsilon}$, where $V_{m}^{0}(t)=\min _{\theta \in[-\tau, 0]} V_{0}[x(t+\theta)]$, and $V_{m}^{0}(t+\tau)>V_{m}^{0}(t)$ for all $t \geq 0$ while trajectories of (1) stay into the set $\Theta_{m}^{V_{0}} \cap \Omega_{\varepsilon}$. Finally, for (1) there exist two options, either a trajectory exits the set $\Omega_{\varepsilon}$ in a finite time or the set $\Omega_{\varepsilon}$ is forward invariant for $(1)$ and $V_{m}^{0}(t+\tau)>V_{m}^{0}(t)$ for all $t \geq 0$. Therefore, the set $\Omega_{\varepsilon}$ contains a repelling trajectory of (1). Further the results of the theorem follow regarding the value of $\lambda_{0}$.

These results establish the links between different variants of local homogeneity with stable/unstable approximating dynamics (3) and the stability/instability properties of the original system (1), similarly to [14].

Corollary 2. Let the system (1) be $\left(r, \lambda_{0}, f_{0}\right)$-homogeneous for some $r_{i}>0$, $i=\overline{1, n}$, the function $f_{0}: R^{n} \rightarrow \mathbb{R}^{n}$ be continuous and $r$-homogeneous with the degree $d_{0}$ and there exist an r-homogeneous Lyapunov-Razumikhin function $V_{0}: \mathbb{R}^{n} \rightarrow \mathbb{R}_{+}$with the degree $\nu_{0}>\max \left\{0,-d_{0}\right\}$ such that $D^{+} V_{0}(x) f_{0}(x) \leq-\alpha(|x|)$ $\left(D^{-} V_{0}(x) f_{0}(x) \geq \alpha(|x|)\right)$ for all $x \in \mathbb{S}_{r}$ with $\alpha \in \mathcal{K}$. Then all conclusions of Theorem 3 (Theorem 4) hold.

Proof. If $f_{0}: \mathbb{R}^{n} \rightarrow \mathbb{R}^{n}$, then the conditions (i) and (ii) of Theorem 3 can be reduced to $D^{+} V_{0}(x) f_{0}(x) \leq-\alpha(|x|)\left(D^{-} V_{0}(x) f_{0}(x) \geq \alpha(|x|)\right)$ for all $x \in \mathbb{S}_{r}$ since the functions $f_{0}$ and $V_{0}$ are homogeneous.

Similarly to [14], one can use these conditions to detect for (1) the presence of Yakubovich's oscillations [33].

\section{ISS PROPERTY OF TIME-DELAY HOMOGENEOUS SYSTEMS}

Consider the system (1) with inputs:

$$
d x(t) / d t=f\left[x_{t}, u(t)\right], t \geq 0,
$$

where $x \in \mathbb{R}^{n}, x_{t} \in C_{[-\tau, 0]}$ is the state function as before, and $u: \mathbb{R}_{+} \rightarrow \mathbb{R}^{m}$ is an essentially bounded (Lebesgue) measurable input, $\|u\|_{\infty}=e s s \cdot \sup _{t>0}|u(t)|$ (we will denote by $\mathcal{L}_{\infty}$ the set of inputs $u: \mathbb{R}_{+} \rightarrow \mathbb{R}^{m}$ with $\|u\|_{\infty}<+\infty$ ); $f$ : $C_{[-\tau, 0]} \times \mathbb{R}^{m} \rightarrow \mathbb{R}^{n}$ is a continuous function (locally Lipschitz with respect to $x_{t}$ ), $f(0,0)=0$. Under these conditions the system (4) has a unique solution $x\left(t, x_{0}, u\right)$ for any $u \in \mathcal{L}_{\infty}$ and $x_{0} \in C_{[-\tau, 0]}$ defined on some interval $[-\tau, T)$. 
The Lyapunov-Razumikhin theory has been extended to the ISS notion analysis in [34] as follows.

Definition 3. The system (4) is called ISS, if for all $x_{0} \in C_{[-\tau, 0]}$ and $u \in \mathcal{L}_{\infty}$ there exist $\beta \in \mathcal{K} \mathcal{L}$ and $\gamma \in \mathcal{K}$ such that for all $t \geq 0$ :

$$
\left|x\left(t, x_{0}, u\right)\right| \leq \beta\left(\left\|x_{0}\right\|, t\right)+\gamma\left(\|u\|_{\infty}\right) .
$$

Theorem 5. Let for the system (4) there exist a locally Lipschitz continuous ISS Lyapunov-Razumikhin function $V: \mathbb{R}^{n} \rightarrow \mathbb{R}_{+}$, i.e. there are functions $\alpha_{1}, \alpha_{2}, \alpha_{3} \in$ $\mathcal{K}_{\infty}$ and $\gamma, \chi \in \mathcal{K}\left(\gamma(s)>s\right.$ for all $\left.s \in \mathbb{R}_{+}\right)$such that for all $x \in \mathbb{R}^{n}, \varphi \in C_{[-\tau, 0]}$ and $u \in \mathbb{R}^{m}$

$$
\begin{gathered}
\alpha_{1}(|x|) \leq V(x) \leq \alpha_{2}(|x|), \\
\max \left(\max _{\theta \in[-\tau, 0]} V[\varphi(\theta)], \chi(|u|)\right)<\gamma\{V[\varphi(0)]\} \Rightarrow D^{+} V[\varphi(0)] f(\varphi, u) \leq-\alpha_{3}(|\varphi(0)|) .
\end{gathered}
$$

Then the system (4) is ISS.

In $[27,28,29]$ it has been shown that if a nonlinear dynamical system is homogeneous, then it is also ISS with respect to an input (e.g. additive disturbance or measurement noise). A similar link for time-delay system (4) is established in the theorem below. Define $\tilde{f}\left(x_{t}, u\right)=\left[f\left(x_{t}, u\right)^{T} 0_{m}\right]^{T} \in \mathbb{R}^{n+m}$, it is an extended auxiliary vector field for the system (4), where $0_{m}$ is the zero vector with $m$ elements.

Theorem 6. Let the vector field $\tilde{f}$ be homogeneous with the weights $r=\left[r_{1}, \ldots, r_{n}\right]>$ $0, \tilde{r}=\left[\tilde{r}_{1}, \ldots, \tilde{r}_{m}\right]>0$ with a degree $d \geq-r_{\min }, r_{\min }=\min _{1 \leq i \leq n} r_{i}$, i.e.

$$
f\left(\Lambda_{r}(\lambda) x_{t}, \Lambda_{\tilde{r}}(\lambda) u\right)=\lambda^{d} \Lambda_{r}(\lambda) f\left(x_{t}, u\right) \quad \forall \lambda>0 .
$$

Assume that for the system (4) for $u=0$ there exists a continuously differentiable $r$-homogeneous Lyapunov-Razumikhin function $V: \mathbb{R}^{n} \rightarrow \mathbb{R}_{+}, V(0)=0$ with degree $\nu>r_{\max }=\max _{1 \leq i \leq n} r_{i}$ such that:

(i) there exist functions $\alpha, \gamma \in \mathcal{K}$ such that for all $\varphi \in \mathcal{S}_{r}$

$$
\max _{\theta \in[-\tau, 0]} V[\varphi(\theta)]<\gamma\{V[\varphi(0)]\} \Rightarrow D^{+} V[\varphi(0)] f(\varphi, 0) \leq-\alpha(|\varphi(0)|) ;
$$

(ii) there exists function $\gamma^{\prime} \in \mathcal{K}$ such that $\lambda s<\gamma^{\prime}(\lambda s) \leq \lambda \gamma(s)$ for all $s, \lambda \in$ $\mathbb{R}_{+} \backslash\{0\}$.

Then the system (4) is ISS.

Proof. Under the introduced conditions $f\left(\Lambda_{r}(\lambda) x_{t}, 0\right)=\lambda^{d} \Lambda_{r}(\lambda) f\left(x_{t}, 0\right)$ and the system $\dot{x}=f\left(x_{t}, 0\right)$ is globally asymptotically stable by Theorem 1 . Since the function $V$ is homogeneous there are functions $\alpha_{1}(s)=\left[\bar{\sigma}_{r}^{-1}(s)\right]^{\nu} \min _{y \in \mathbb{S}_{r}} V(y)$, $\alpha_{2}(s)=\left[\underline{\sigma}_{r}^{-1}(s)\right]^{\nu} \max _{y \in \mathbb{S}_{r}} V(y)$ such that $\alpha_{1}(|x|) \leq V(x) \leq \alpha_{2}(|x|)$ for all $x \in \mathbb{R}^{n}$. 
It has been shown in the proof of Theorem 1 that for all $\varphi \in \mathcal{S}_{r}$

$$
\begin{gathered}
\max _{\theta \in[-\tau, 0]} V[\varphi(\theta)]<\gamma\{V[\varphi(0)]\} \Rightarrow D^{+} V[\varphi(0)] f(\varphi, 0) \leq-a, \\
\left|D^{+} V[\varphi(0)]\right| \leq b
\end{gathered}
$$

for some $a>0$ and $b>0$. Due to homogeneity of $\tilde{f}$ and continuity of $f$ with respect to $u$ we have

$$
\begin{gathered}
|f(\varphi, u)-f(\varphi, 0)| \leq \sigma(|u|) \quad \forall \varphi \in \mathcal{S}_{r}, \\
\sigma(s)=c \begin{cases}s^{\varrho_{\min }} & \text { if } s \leq 1 \\
s^{\varrho_{\max }} & \text { if } s>1\end{cases}
\end{gathered}
$$

for some $c>0$ and $\varrho_{\max } \geq \varrho_{\min }>0$.

Below we will use the coordinate transformation $\phi=\Lambda_{r}\left(\|\phi\|_{r}\right) \varphi$, which connects any $\phi \in C_{[-\tau, 0]} \backslash\{0\}$ with the corresponding point $\varphi \in \mathcal{S}_{r}$. For the input $u$ we will use the transformation $u=\Lambda_{\tilde{r}}\left(\|\phi\|_{r}\right) \tilde{u}$, where $\tilde{u} \in \mathbb{R}^{m}$ and

$$
|\tilde{u}| \leq \rho\left(\|\phi\|_{r}\right)|u|, \rho(s)=\left\{\begin{array}{ll}
s^{-\tilde{r}_{\max }} & \text { if } s \leq 1 \\
s^{-\tilde{r}_{\min }} & \text { if } s>1
\end{array},\right.
$$

where $\tilde{r}_{\max }=\max _{1 \leq j \leq m} \tilde{r}_{j}$ and $\tilde{r}_{\min }=\min _{1 \leq j \leq m} \tilde{r}_{j}$. Now let us consider the time derivative of the Lyapunov function $V$ computed for the system (4) for all $\phi \in C_{[-\tau, 0]}$ and $u \in \mathbb{R}^{m}$ :

$$
\begin{aligned}
D^{+} V[\phi(0)] f(\phi, u) & =\|\phi\|_{r}^{\nu+d} D^{+} V[\varphi(0)] f(\varphi, \tilde{u}) \\
& =\|\phi\|_{r}^{\nu+d} D^{+} V[\varphi(0)] f(\varphi, 0)+\|\phi\|_{r}^{\nu+d} D^{+} V[\varphi(0)]\{f(\varphi, \tilde{u})-f(\varphi, 0)\} .
\end{aligned}
$$

Assume that $\max _{\theta \in[-\tau, 0]} V[\varphi(\theta)]<\gamma\{V[\varphi(0)]\}$ or equivalently (due to homogeneity of $V$, see the proof of Theorem 1) $\max _{\theta \in[-\tau, 0]} V[\phi(\theta)]<\gamma^{\prime}\{V[\phi(0)]\}$, then

$$
\begin{aligned}
D^{+} V[\phi(0)] f(\phi, u) & \leq-a\|\phi\|_{r}^{\nu+d}+b\|\phi\|_{r}^{\nu+d} \sigma(|\tilde{u}|) \\
& \leq-a\|\phi\|_{r}^{\nu+d}+b\|\phi\|_{r}^{\nu+d} \kappa\left(\|\phi\|_{r}\right) \sigma(|u|),
\end{aligned}
$$

where

$$
\kappa(s)=\left\{\begin{array}{ll}
s^{-\tilde{r}_{\max } \varrho_{\min }} & \text { if } s \leq 1 \\
s^{-\tilde{r}_{\min } \varrho_{\max }} & \text { if } s>1
\end{array} .\right.
$$

Therefore if $-\tilde{r}_{\min } \varrho_{\max }<0$, which is equivalent to $\tilde{r}_{\min }>0$, then $\eta(s)=\kappa(s)^{-1} \in$ $\mathcal{K}_{\infty}$ and for $|u| \leq \sigma^{-1}\left[a /(2 b) \eta\left(\|\phi\|_{r}\right)\right]$ we have $D^{+} V[\phi(0)] f(\phi, u) \leq-0.5 a\|\phi\|_{r}^{\nu+d}$. Since

$\alpha_{1}(\| \phi||)=\alpha_{1}\left(\max _{\theta \in[-\tau, 0]}|\phi(\theta)|\right)=\max _{\theta \in[-\tau, 0]} \alpha_{1}(|\phi(\theta)|) \leq \max _{\theta \in[-\tau, 0]} V(\phi(\theta))<\gamma^{\prime}\{V[\phi(0)]\}$, 
for $\chi(s)=\alpha_{1} \circ \underline{\rho}_{r} \circ \eta^{-1}[2 b / a \sigma(s)]$ and $\alpha_{3}(s)=0.5 a s^{\nu+d}$ we obtain the property

$\max \left(\max _{\theta \in[-\tau, 0]} V[\varphi(\theta)], \chi(|u|)\right)<\gamma^{\prime}\{V[\varphi(0)]\} \Rightarrow D^{+} V[\varphi(0)] f(\varphi, u) \leq-\alpha_{3}\left(|\varphi(0)|_{r}\right)$, which implies ISS.

The result of Theorem 6 says that if the conditions of Theorem 1 are satisfied for the case $u=0$ for the system (4), then it is ISS. This conclusion highlights an additional importance of the introduced homogeneity concept for time-delay systems: under additional algebraic restrictions on the system equations and its Lyapunov-Razumikhin function (homogeneity) we gain the system robustness.

Corollary 3. Let a locally Lipschitz continuous function $f_{0}: C_{[-\tau, 0]} \rightarrow \mathbb{R}^{n}$ be $r$ homogeneous with a degree $d$ and admit a continuously differentiable $r$-homogeneous Lyapunov-Razumikhin function $V: \mathbb{R}^{n} \rightarrow \mathbb{R}_{+}, V(0)=0$ with degree $\nu>r_{\max }$.

If $f\left(x_{t}, u\right)=f_{0}\left(x_{t}\right)+u$, i.e. $u$ is an additive disturbance, then the system (4) is ISS for $d>-r_{\min }$.

If $f\left(x_{t}, u\right)=f_{0}\left(x_{t}+u\right)$, i.e. $u$ is a measurement noise, then the system (4) is ISS.

Proof. Take $\tilde{r}=r+\nu$ and $\tilde{r}=r$ for the additive disturbance and measurement noise cases respectively.

\section{EXAmples}

In this section we will consider a homogeneous system with zero degree, two academic examples of locally homogeneous time-delay systems (to illustrate various aspects of theorems 3 and 4) and one model from biology of blood cell production.

6.1. A homogeneous time-delay system with degree $d=0$. Consider the system

$$
\dot{x}(t)=-2 x(t)+\frac{x^{2}(t)+x^{2}(t-\tau)}{\max \{|x(t)|,|x(t-\tau)|\}},
$$

where $x(t) \in \mathbb{R}, \tau>0$ is a fixed time delay. Applying the Lyapunov-Razumikhin approach with $V(x)=0.5 x^{2}$ it is straightforward to show that the system is stable. The system is homogeneous for $r=1$ and $d=0$. According to Proposition 1, if $x\left(t, x_{0}\right)$ is a solution of this system with initial condition $x_{0} \in C_{[-\tau, 0]}$, then $y\left(t, y_{0}\right)=\lambda x\left(t, x_{0}\right)$ is the system solution with initial condition $y_{0}=\lambda x_{0}$ for any $\lambda>0$. For $\tau=2$, the system trajectory $x\left(t, x_{0}\right)$ with $x_{0}(s)=e^{s}, s \in[-\tau, 0]$ and the system trajectory $y\left(t, y_{0}\right)=2 x\left(t, x_{0}\right)$ for $y_{0}(s)=2 e^{s}, s \in[-\tau, 0]$ are shown in Fig. 1. The results of simulation confirm the scaling property established in Proposition 1. 


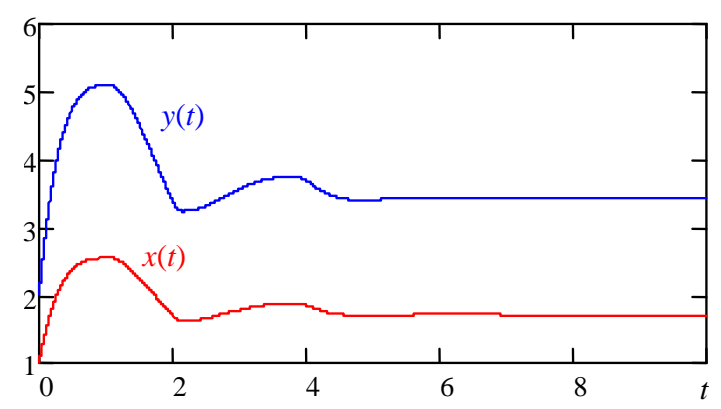

FigURE 1. Trajectories of a homogeneous system with degree zero

6.2. A globally stable system. Consider the system:

$$
\begin{aligned}
& \dot{x}_{1}(t)=-x_{1}(t)+x_{2}(t)-x_{1}^{3}(t) ; \\
& \dot{x}_{2}(t)=-k x_{2}(t)-x_{1}(t)+a x_{2}(t-\tau)-x_{2}^{3}(t),
\end{aligned}
$$

where $k, a$ are some positive parameters, $\tau>0$ is a fixed time delay. This system is locally homogeneous in the bi-limit:

$$
\begin{gathered}
\lambda_{1}=0, r_{1}=\left[\begin{array}{ll}
0.5 & 0.5
\end{array}\right] \\
f_{1}\left(x_{\tau}\right)=\left[\begin{array}{c}
-x_{1}(t)+x_{2}(t), \quad-k x_{2}(t)-x_{1}(t)+a x_{2}(t-\tau) \\
]^{T}, \\
d_{1}=0, V_{1}(x)=x_{1}^{2}(t)+x_{2}^{2}(t), v_{1}=1
\end{array}\right. \\
\lambda_{2}=+\infty, r_{2}=\left[\begin{array}{ll}
1 & 1
\end{array}\right], f_{2}\left(x_{\tau}\right)=\left[\begin{array}{ll}
-x_{1}^{3}(t), & -x_{2}^{3}(t)
\end{array}\right]^{T}, \\
d_{2}=2, V_{2}(x)=x_{1}^{2}(t)+x_{2}^{2}(t), v_{2}=2 .
\end{gathered}
$$

Straightforward calculations show that

$$
\begin{aligned}
\dot{V}_{1} & =2\left[-x_{1}^{2}(t)-k x_{2}^{2}(t)+a x_{2}(t) x_{2}(t-\tau)\right] \\
& \leq 2\left[-x_{1}^{2}(t)-(k-0.5 a) x_{2}^{2}(t)\right]+a x_{2}^{2}(t-\tau) \\
& \leq-2 \min \{1, k-0.5 a\} V_{1}(t)+a V_{1}(t-\tau) .
\end{aligned}
$$

Then

$$
\begin{gathered}
\max _{\theta \in[-\tau, 0]} V_{1}(x(t+\theta))<a^{-1} \min \{1, k-0.5 a\} V_{1}(t) \Rightarrow \\
\dot{V}_{1} \leq-2 \min \{1, k-0.5 a\} V_{1},
\end{gathered}
$$

and if $a^{-1} \min \{1, k-0.5 a\}>1$ the system (3) at $\lambda_{1}=0$ is asymptotically stable, that according to Theorem 3 implies local asymptotic stability of the system around 


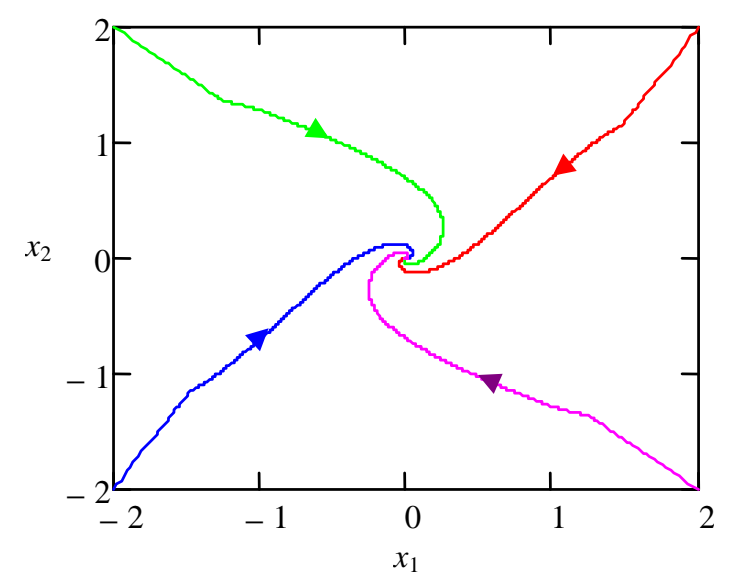

Figure 2. The results of simulation for a stable bi-limit homogeneous system

the origin. Obviously for $\lambda_{2}=+\infty$ the system (3) is also asymptotically stable that ensures global convergence of the trajectories to a vicinity of the origin. Note that approximate behavior of the system in this case is defined by the time-delay free dynamics (Corollary 2). The results of simulation for $k=1, a=0.5, \tau=0.1$ presented in Fig. 2 illustrate that actually the system is globally asymptotically stable.

6.3. An oscillating system. Consider another planar system:

$$
\begin{aligned}
& \dot{x}_{1}(t)=a x_{1}(t)-x_{2}(t-\tau)-x_{1}^{3}(t)+x_{1}^{2}(t) ; \\
& \dot{x}_{2}(t)=k x_{2}(t)+x_{1}(t-\tau)-x_{1}^{2}(t) x_{2}(t)+x_{1}(t-\tau) x_{2}(t),
\end{aligned}
$$

where $k>1.5, a>1.5$ are some positive parameters, $\tau>0$ is a fixed time delay. The system is locally homogeneous in the bi-limit:

$$
\begin{gathered}
\lambda_{1}=0, r_{1}=\left[\begin{array}{ll}
1 & 1
\end{array}\right], \\
f_{1}\left(x_{\tau}\right)=\left[\begin{array}{ll}
a x_{1}(t)-x_{2}(t-\tau) & k x_{2}(t)+x_{1}(t-\tau)
\end{array}\right]^{T}, \\
d_{1}=0, V_{1}(x)=x_{1}^{2}(t)+x_{2}^{2}(t), v_{1}=2 ; \\
\lambda_{2}=+\infty, r_{2}=\left[\begin{array}{ll}
0.51 & 1, f_{2}\left(x_{\tau}\right)=\left[\begin{array}{ll}
-x_{1}^{3}(t) & -x_{1}^{2}(t) x_{2}(t)
\end{array}\right]^{T}, \\
d_{2}=1, V_{2}(x)=x_{1}^{2}(t)+\left|x_{2}(t)\right|, v_{2}=1 .
\end{array}\right.
\end{gathered}
$$




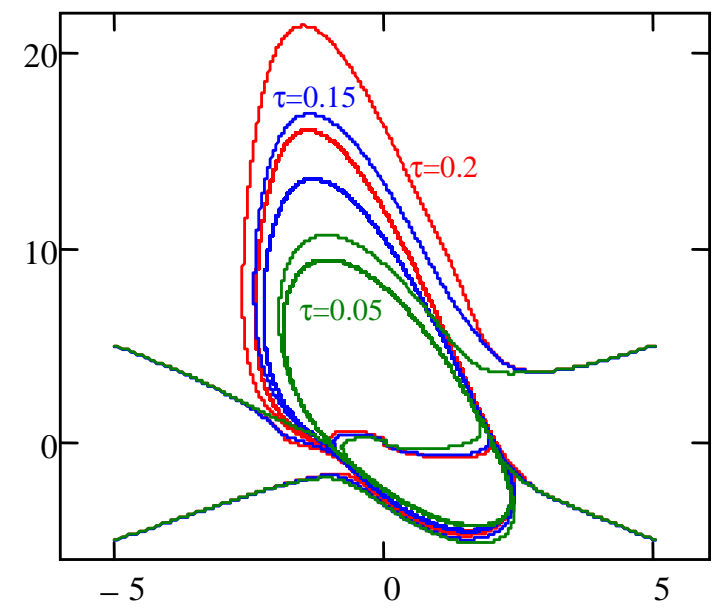

Figure 3. Trajectories of an oscillating bi-limit homogeneous system

For $\lambda_{1}=0$ we have:

$$
\begin{aligned}
\dot{V}_{1}(t) & =2 x_{1}(t)\left[a x_{1}(t)-x_{2}(t-\tau)\right]+2 x_{2}(t)\left[k x_{2}(t)+x_{1}(t-\tau)\right] \\
& =2 a x_{1}^{2}(t)-2 x_{1}(t) x_{2}(t-\tau)+2 k x_{2}^{2}(t)+2 x_{2}(t) x_{1}(t-\tau) \\
& \geq[2 a-1] x_{1}^{2}(t)-x_{2}^{2}(t-\tau)+[2 k-1] x_{2}^{2}(t)-x_{1}^{2}(t-\tau) \\
& \geq \mu V_{1}(t)-V_{1}(t-\tau), \mu=2 \min \{a, k\}-1 .
\end{aligned}
$$

Then $V_{1}(t) \geq V_{1}(t-\tau)$ into the set $P_{M}^{V_{1}}$ and $\dot{V}_{1}(t) \geq(\mu-1) V_{1}(t-\tau)>0$ since $\mu>1$. Therefore, the conditions of Theorem 4 are satisfied and the system is unstable at the origin. At $\lambda_{2}=+\infty$ the system has the same approximation dynamics as in the previous example, therefore it has globally bounded trajectories. Since the system has the single equilibrium at the origin, then under this conditions it is oscillating in the sense of Yakubovich [33]. This conclusion is confirmed by the results of numerical simulation presented in Fig. 3 for different values of $\tau$.

6.4. Blood cell production model. The process of blood cell production is based on differentiation of hematopoietic stem cells located in the bone marrow. Mathematical modeling of this process has a long research history (see [35, 36, 37] and references therein). A model of the blood cell production can be applied for instance to study of chronic myelogenous leukemia [35].

In $[35,36]$ the following model has been analyzed:

$$
\begin{aligned}
& \dot{P}(t)=-\gamma P(t)+\beta[N(t)] N(t)-e^{-\gamma \tau} \beta[N(t-\tau)] N(t-\tau), \\
& \dot{N}(t)=-\delta N(t)-\beta[N(t)] N(t)+2 e^{-\gamma \tau} \beta[N(t-\tau)] N(t-\tau),
\end{aligned}
$$




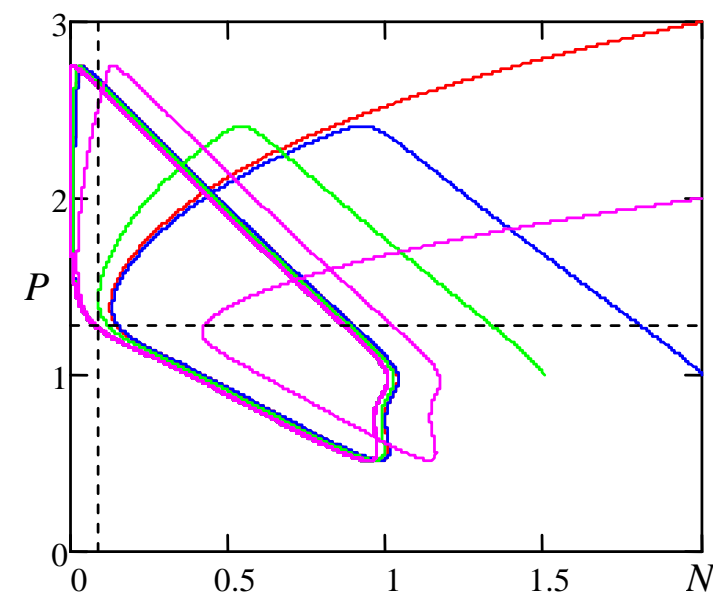

Figure 4. Trajectories of the model from $[35,36]$

where $P \in \mathbb{R}_{+}$and $N \in \mathbb{R}_{+}$are concentrations of proliferating and nonprolifirating cells, $\tau>0$ defines an average duration of the cell cycle, $\gamma, \delta>0$ define the apoptosis and differentiation rates for populations $P$ and $N$ respectively, and $\beta: \mathbb{R}_{+} \rightarrow \mathbb{R}_{+}$ is a nonlinear function characterizing proliferation rate and interrelations between these populations such that

$$
\lim _{N \rightarrow+\infty} \beta(N)=0, \lim _{N \rightarrow+\infty} N \beta(N)=0 .
$$

An example is the Hill function $\beta(N)=\frac{a}{b+N^{h}}$ with $a>0, b>0$ and $h>1$. The conditions of existence of equilibriums (a positive one and at the origin) and their local stability have been investigated in the papers $[35,36]$ (see also references therein).

In this work we would like to establish global stability of this model using the homogeneity theory developed here. For $r=\left[\begin{array}{ll}1 & 1\end{array}\right]$ the system has the following approximating dynamics at infinity (due to properties of the Hill function)

$$
\begin{aligned}
& \dot{P}(t)=-\gamma P(t), \\
& \dot{N}(t)=-\delta N(t),
\end{aligned}
$$

which is clearly delay-invariant and globally asymptotically stable, thus by Theorem 3 this model has bounded trajectories. Next, applying analysis of linearization of the system at the equilibriums (it is done in $[35,36]$ ), if in both equilibriums the system has unstable linearization, then according to the proven global boundedness and the Yakubovich's oscillation theory [33] the system is oscillating. The results of the system simulation (with the parameters $\gamma=0.2, \delta=0.05, \tau=1, a=1.77$, $b=1$ and $h=12$ calculated in $[35,36])$ are shown in Fig 4. 


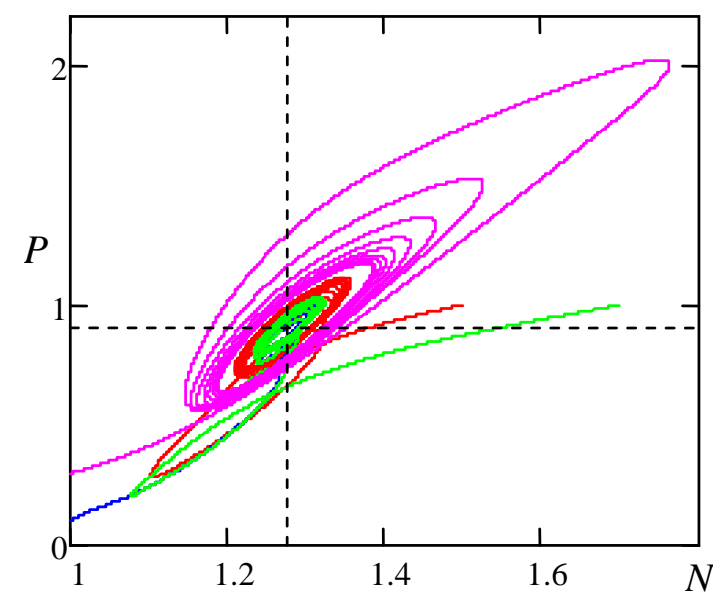

Figure 5. Trajectories of the model from [37]

Following [37] consider another model of the blood cell production, where the nonlinear coupling function $\beta$ depends on the total concentration of proliferating and nonprolifirating cells $S(t)=N(t)+P(t)$ :

$$
\begin{aligned}
\dot{S}(t) & =-\delta S(t)+e^{-\delta \tau} \beta[S(t-\tau)] N(t-\tau), \\
\dot{N}(t) & =-\delta N(t)-\beta[S(t)] N(t)+2 e^{-\delta \tau} \beta[S(t-\tau)] N(t-\tau),
\end{aligned}
$$

all parameters have the same meaning as before.

If we would try to calculate approximation at infinity of this system with the weights $r=\left[\begin{array}{ll}1 & 1\end{array}\right]$, in order to analyze its global stability using Theorem 3 , we cannot arrive to a conclusion since this approximation does not exist. Indeed, according to definition of local homogeneity, the limit for $\lambda_{0}=+\infty$ should be uniform on $\mathcal{S}_{r}$, for the term $\beta[S] N$ the point where $S=0$ is singular and a uniform on $\mathcal{S}_{r}$ limit does not exist. However, in [37] it is shown that this system has only nonnegative solutions. In fact it is possible to show that the cone $K=\left\{(S, N) \in \mathbb{R}_{+}^{2}: S \geq \epsilon N\right\}$ for some $0<\epsilon<+\infty$ is forward invariant for the system. Indeed, define $y=S-\epsilon N$, then

$$
\dot{y}(t)=-\delta y(t)+\epsilon \beta[S(t)] N(t)+(1-2 \epsilon) e^{-\delta \tau} \beta[S(t-\tau)] N(t-\tau)
$$

and on the line $y=0$ we have $\dot{y} \geq 0$ provided that $\epsilon \leq 0.5$. Therefore, if $y(0) \geq 0$, then $y(t) \geq 0$ for all $t \geq 0$, that is equivalent to forward invariance of $K$. In this case, in order to calculate the system homogeneous approximation at infinity on $K$, it is sufficient to compute a limit uniformly on $\mathcal{S}_{r} \cap K$, where it exists and the system has the same approximation dynamics at infinity (5). For the parameters $\delta=0.05, \tau=5, a=1.77, b=1$ and $h=12[37]$ the system has unstable linearization in both equilibriums, therefore following [33] it is oscillating in the sense of Yakubovich. The corresponding trajectories are shown in Fig. 5. 


\section{Conclusions}

The homogeneity notion is extended to time-delay systems (nonlinear differential equations with functional arguments). It is proven that a local stability notion for homogeneous time-delay systems with $d=0$ holds globally, that simplifies analysis of such a type of systems applying linearization at the origin, for instance. The sufficient conditions for stability/instability of homogeneous systems are presented. These conditions are based on the Razumikhin stability arguments. It is shown on a counterexample that development of the Lyapunov-Krasovskii approach is tricky for a generic homogeneous case. It is also shown that if a homogeneous system has a homogeneous Lyapunov-Razumikhin function, then under a mild structural condition (dealing with degree of homogeneity) it is ISS. The definition of local homogeneity is proposed, relations between stability/instability of the locally approximating dynamics and the original system are established. Efficiency of the proposed approach is demonstrated on examples.

\section{ACKNOWLEDGMENT}

The authors would like to thank the anonymous reviewers for their valuable comments and suggestions.

\section{References}

[1] L. Rothschild and E. Stein, "Hypoelliptic differential operators and nilpotent groups," Acta Mathematica, vol. 137, pp. 247-320, 1976.

[2] V. Andrieu, L. Praly, and A. Astolfi, "Homogeneous approximation, recursive observer design, and output feedback," SIAM J. Control Optimization, vol. 47, no. 4, pp. 1814-1850, 2008.

[3] A. Bacciotti and L. Rosier, Liapunov Functions and Stability in Control Theory, vol. 267 of Lecture Notes in Control and Inform. Sci. Berlin: Springer, 2001.

[4] H. Hermes, Differential Equations: Stability and Control, vol. 109 of Lecture Notes in Pure Appl. Math., ch. Homogeneous coordinates and continuous asymptotically stabilizing feedback controls, pp. 249-260. Marcel Dekker, 1991.

[5] Y. Hong, "Finite-time stabilization and stabilizability of a class of controllable systems," Systems\&Control Lett., vol. 46, pp. 231-236, 2002.

[6] L. Rosier, "Homogeneous Lyapunov function for homogeneous continuous vector field," Systems\&Control Lett., vol. 19, pp. 467-473, 1992.

[7] H. Hermes, "Nilpotent and high-order approximations of vector field systems," SIAM Review, vol. 33, no. 2, pp. 238-264, 1991.

[8] T. Ménard, E. Moulay, and W. Perruquetti, "Homogeneous approximations and local observer design," ESAIM: Control, Optimization and Calculus of Variations, vol. 19, no. 3, pp. 906$929,2013$.

[9] S. Bhat and D. Bernstein, "Geometric homogeneity with applications to finite-time stability," Mathematics of Control, Signals and Systems, vol. 17, pp. 101-127, 2005.

[10] L. Grüne, "Homogeneous state feedback stabilization of homogeneous systems," SIAM J. Control Optimization, vol. 38, no. 4, pp. 1288-1314, 2000. 
[11] M. Kawski, Homogeneous feedback stabilization, vol. 7 of Progress in systems and control theory: New trends in systems theory. Birkhäuser, 1991.

[12] E. Moulay and W. Perruquetti, "Finite time stability and stabilization of a class of continuous systems," J. Mathematical Analysis Applications, vol. 323, no. 2, pp. 1430-1443, 2006.

[13] R. Sepulchre and D. Aeyels, "Stabilizability does not imply homogeneous stabilizability for controllable systems," SIAM J. Control Optimization, vol. 34, no. 5, pp. 1798-1813, 1996.

[14] D. Efimov and W. Perruquetti, "Oscillations conditions in homogenous systems," in Proc. NOLCOS'10, (Bologna), pp. 1379-1384, 2010.

[15] J. Chiasson and J. Loiseau, eds., Applications of Time Delay Systems, vol. 352 of Lecture Notes in Control and Information Sciences. Springer, 2007.

[16] J.-P. Richard, "Time-delay systems: an overview of some recent advances and open problems," Automatica, vol. 39, pp. 1667-1694, 2003.

[17] K. Gu, K. Kharitonov, and J. Chen, Stability of Time-Delay Systems. Control Engineering, Boston: Birkhäuser, 2003.

[18] J. Hale, Theory of Functional Differential Equations. Springer-Verlag, 1977.

[19] V. Kolmanovsky and V. Nosov, Stability of functional differential equations. San Diego: CA: Academic, 1986.

[20] H. Gao, T. Chen, and A. Chai, "Passivity and passification for networked control systems," SIAM J. Control Optim., vol. 46, no. 4, pp. 1299-1322, 2007.

[21] A. Kruszewski, W. Jiang, E. Fridman, J.-P. Richard, and A. Toguyeni, "A switched system approach to exponential stabilization through communication network," IEEE Transactions on Control Systems Technology, vol. 20, no. 4, pp. 887-900, 2012.

[22] E. Fridman, "A refined input delay approach to sampled-data control," Automatica, vol. 46, pp. 421-427, 2010.

[23] A. Aleksandrov and A. Zhabko, "On the asymptotic stability of solutions of nonlinear systems with delay," Siberian Mathematical Journal, vol. 53, no. 3, pp. 393-403, 2012.

[24] F. Asl and A. Ulsoy, "Analytical solution of a system of homogeneous delay differential equations via the Lambert function," in Proc. American Control Conference, (Chicago), pp. 2496$2500,2000$.

[25] V. Bokharaie, O. Mason, and M. Verwoerd, "D-stability and delay-independent stability of homogeneous cooperative systems," IEEE Trans. Automatic Control, vol. 55, no. 12, pp. 2882$2885,2010$.

[26] J. Dublik, "Asymptotic equilibrium for homogeneous delay linear differential equations with l-perturbation term," Nonlinear Analysis, Theory, Methods \& Applicarions, vol. 30, no. 6, pp. 3927-3933, 1997.

[27] E. Ryan, "Universal stabilization of a class of nonlinear systems with homogeneous vector fields," Systems \& Control Letters, vol. 26, pp. 177-184, 1995.

[28] Y. Hong, " $\mathrm{H}_{\infty}$ control, stabilization, and input-output stability of nonlinear systems with homogeneous properties," Automatica, vol. 37, no. 7, pp. 819-829, 2001.

[29] E. Bernuau, A. Polyakov, D. Efimov, and W. Perruquetti, "On iss and iiss properties of homogeneous systems," in Proc. European Control Conference (ECC) 2013, (Zürich), 2013.

[30] E. Bernuau, A. Polyakov, D. Efimov, and W. Perruquetti, "Verification of iss, iiss and ioss properties applying weighted homogeneity," Systems \& Control Letters, vol. 62, no. 12, pp. 1159-1167, 2013. 
[31] J. Haddock and Y. Ko, "Lyapunov-Razumikhin functions and an instability theorem for autonomous functional differential equations with finite delay," Rocky Mtn. J. Math., vol. 25, pp. 261-267, 1995.

[32] J. Haddock and J. Zhao, "Instability for autonomous and periodic functional differential equations with finite delay," Funkcialaj Ekvacioj, vol. 39, pp. 553-570, 1996.

[33] D. Efimov and A. Fradkov, "Oscillatority conditions for nonlinear systems with delays," Journal of Applied Mathematics, pp. 1-12, 2007. Article ID 72561.

[34] A. R. Teel, "Connections between Razumikhin-type theorems and the ISS nonlinear small gain theorem," IEEE Trans. Automat. Control, vol. 43, no. 7, pp. 960-964, 1998.

[35] L. Pujo-Menjouet and M. Mackey, "Contribution to the study of periodic chronic myelogenous leukemia," C. R. Biologies, vol. 327, pp. 235-244, 2004.

[36] L. Pujo-Menjouet, S. Bernard, and M. Mackey, "Long period oscillations in a g0 model of hematopoietic stem cells," SIAM J. Appl. Dyn. Systems, vol. 4, no. 2, pp. 312-332, 2005.

[37] F. Crauste, "Global Asymptotic Stability and Hopf Bifurcation for a Blood Cell Production Model," Mathematical Biosciences and Engineering, vol. 3, no. 2, pp. 325-346, 2006. 